\title{
A Haar Wavelet Method for Angularly Discretising the Boltzmann Transport Equation
}

Babatunde J. Adigun $^{\mathrm{a}}$, Andrew G. Buchan ${ }^{\mathrm{b}, *}$, Alexandros Adam ${ }^{\mathrm{a}}$, Steven Dargaville ${ }^{\mathrm{a}}$, Mark A. Goffin ${ }^{\mathrm{a}}$, Christopher C. Pain ${ }^{\mathrm{a}}$

\author{
${ }^{a}$ Applied Modelling and Computation Group \\ Department of Earth Science and Engineering \\ Imperial College London, $S W^{\text {r }} 2 \mathrm{2} \mathrm{AZ}, \mathrm{UK}$ \\ ${ }^{b}$ Division of Engineering Science \\ School of Engineering and Material Science \\ Queen Mary University of London, E1 4NS, UK
}

\begin{abstract}
A novel, hierarchical Haar wavelet basis is introduced and used to discretise the angular dimension of the Boltzmann transport equation. This is used in conjunction with a finite element subgrid scale method. This combination is then validated using two steady-state radiation transport problems, namely a 2D dogleg-duct shielding problem and the 2D C5MOX OECD/NEA benchmark. It is shown that the scheme has many similarities to a traditional equal weighted discrete ordinates $\left(S_{n}\right)$ angular discretisation, but the strong motivation for our hierarchical Haar wavelet method is the potential for adapting in angle in a simple fashion through elimination of redundant wavelets. Initial investigations of this adaptive approach are presented for a shielding and criticality eigenvalue example. It is shown that a $60 \%$ reduction in the number of angles needed on most spatial nodes - and rising up to $90 \%$ on nodes located in high streaming areas - can be attained without adversely affecting the accuracy of the solution.
\end{abstract}

Keywords: Radiation Transport; Boltzmann Transport Equation; Haar Wavelet; C5MOX; Adaptivity

\footnotetext{
${ }^{*}$ Corresponding author

Email address: andrew.buchan@imperial.ac.uk (Andrew G. Buchan )
} 


\section{Introduction}

Modern wavelet research was pioneered by the likes of Morlet, Grossman, and Daubechies $[1,2]$. The research efforts of the former two in signal processing led to the creation of a wavelet transform method that allowed the representation of information localised simultaneously in time and frequency, a feat otherwise not possible using Fourier Transforms [1]. The wavelet transform method was also unique in that the wavelet used as the analysing functions were not limited to a particular type. This spurred the proposal of several wavelet types (or families) differing in form from constant functions to polynomials and exponentials [3, 4]. Some examples of wavelet families include the Legendre, Morlet, Gaussian, Shannon, Haar, and Daubechies wavelets [5].

While the proposed wavelet families differed individually, they all shared the property of being zero everywhere except on a small interval. Such functions with zero values everywhere outside their closed and bounded intervals were said to be compactly supported [5]. Daubechies' work would later produce a ground-breaking paper on compactly supported wavelets, proving that it was possible to develop wavelets with desired properties tailored to specific applications [2]. The research work which ensued led to the development of the multiresolution analysis (MRA) which enabled a hierarchical wavelet reconstruction of a signal in layers of increasing resolution. The second-generation wavelet transform was later developed to address some of the deficiencies of traditional wavelets, allowing them to be used in representing functions on arbitrary domains [4].

Before long, wavelets became a very useful tool in a number of fields such as image/data compression and computer graphics applications, and over time, expanded into a number of numerical analysis fields $[5,6,7]$. In solving ordinary differential equations and partial differential equations, wavelets have been applied for the Navier-Stokes equation [8, 9, 10] and other parabolic $[11,12,13]$ and hyperbolic equations $[14,15,16,17]$. More recently, wavelets

have been applied in solving the Boltzmann Transport Equation (BTE) [6, 18, 19, 20, 21, 22]. Schroder and Sweldens [4] spearheaded the construction of wavelets on the sphere to represent spherical functions. Some other works showing the different applications of wavelets on the sphere include Freeden and Windheuser [23], Demanet et al. [24], Antoine and Vandergheynst [25], and Buchan et al. [26]. 
This article presents a new approach for representing the direction of neutron particle travel through a Haar wavelet discretisation of the angular dimension of the BTE. Related works include that of Buchan et al. who applied linear and quadratic octahedral wavelets [6] as well as self-adaptive spherical wavelets [26] on the sphere to represent the angular flux of the BTE. Cao et al. used a Daubechies' double wavelets expansion in solving the angular domain of the neutron transport equation [19]. Recently, a two-dimensional Haar wavelet collocation method has been used by Patra and Ray to solve the neutron transport [27] and point kinetics equation [28]. However, this is the first time the angular variable of the neutron flux has been discretised through a dual Haar wavelet expansion on the sphere combined with a subgrid scale (SGS) finite element method (FEM) that enables an efficient solution method.

The dual Haar wavelet discretisation can be shown to generate identical discretisations to the $S_{n}$ method when particular weights and directions are used. However a strong motivation for pursuing this Haar wavelet approach is that its inherent properties of using compactly supported functions within a hierarchical expansion scheme allow the incorporation of angular adaptivity into the discretisation. Compactly supported functions locally resolve the problem meaning that only those that contribute most to the solution need to be retained. Redundant wavelet functions that contribute little to the solution can be eliminated from the calculation and this can be achieved in a consistent manner through the use of hierarchical expansions, as shown in [26]. As such, resolution can be focused on regions of angle where it is needed most using fewer functions than traditional approaches to enhance computational efficiency. This Haar approach also has advantages over other similar wavelet schemes, e.g. [26], as it leads to more sparse discretised matrix systems that can be utilized to improve solving times. It also bridges the gap beteen $S_{n}$ and adaptivity in angle, although other important works in this area include that of Adams and Larsen [29], Jessee et al. [30], Ragusa and Wang [31].

The aim of this article is to lay the foundations by developing the Haar wavelet angular discretisation for the resolution of the BTE. It will show that the Haar wavelet method can produce solutions that are identical to certain $S_{n}$ quadrature schemes. However it will also show these quadrature rules are not necessarily optimal in comparison to other $S_{n}$ methods. The most important component for the Haar wavelets' use in neutron transport is therefore their potential to adapt in angle, which may substantially reduce problem size and decrease computational solving times. For certain problem types angular adaptivity could exceed standard $S_{n}$ methods when constant and high resolution quadrature sets are used. To lay the foundations this paper focuses on two developments in addition to the Haar wavelet discretisation. Firstly it will develop the angular Haar wavelet method within a spatial discretisation scheme, suitable for the BTE, that can be solved efficiently without a sweep based solver. This is vital as the Haar wavelets will introduce coupling of angular moments 
through the streaming operator and sweep-based $S_{n}$ like solvers can no longer be employed. Secondly we will demonstrate the potential of adaptivity by filtering redundant wavelets from their solution. This aims to highlight the powers of adaptivity and indicate the reduction in problem size that may be achieved. A second article will follow the developments here showing how the model and solver can be adapted to enable self-adaptivity that produces highly efficient solutions in angular resolution.

The sections of this article are set out as follows. In section 2 the BTE is introduced and the discretisation of the space and angle dimensions are given. In section 3, the MRA is introduced and the general wavelet MRA is applied to the Haar wavelet family in capturing the angular dependence of the BTE. In section 4, two numerical examples are presented. These are specifically chosen to demonstrate the capability of the Haar approach over a range of radiation transport conditions and to illustrate the potential gains from using adaptivity with Haar wavelets in this framework. Finally, section 5 completes the paper with a conclusion on the findings and proposed future works.

\section{Discretisation of the Boltzmann transport equation}

The following sections describe the BTE and provide an overview of the space-angle discretisation methods used in this article.

\subsection{The Boltzmann Transport Equation}

The BTE governs the conservation of neutral particle transport within its surrounding medium. For fixed source problems, the first order time-independent multi-group equations are given as $[32,33]$

$$
\begin{array}{r}
\hat{\mathbf{\Omega}} \cdot \nabla \psi_{g}(\boldsymbol{r}, \hat{\mathbf{\Omega}})+\Sigma_{t, g}(\boldsymbol{r}) \psi_{g}(\boldsymbol{r}, \hat{\mathbf{\Omega}})=\int_{4 \pi} \sum_{g^{\prime}=1}^{G} \Sigma_{s, g^{\prime} \rightarrow g}\left(\boldsymbol{r}, \hat{\boldsymbol{\Omega}}^{\prime} \rightarrow \hat{\mathbf{\Omega}}\right) \psi_{g^{\prime}}\left(\boldsymbol{r}, \hat{\boldsymbol{\Omega}}^{\prime}\right) \mathrm{d} \hat{\Omega}^{\prime}+S_{g^{\prime}}(\boldsymbol{r}, \hat{\mathbf{\Omega}}), \\
\forall g \in\{1,2,3 \ldots, G\},
\end{array}
$$

where $\mathrm{G}$ energy groups are represented and the subscript $\mathrm{g}$ denotes each energy group. The angular flux, $\psi_{g}(\boldsymbol{r}, \hat{\boldsymbol{\Omega}})$, is defined over a five dimensional solution space; three of which are 
in the spatial direction $\boldsymbol{r}$, and two of which are in the direction of travel or angular domain $\hat{\mathbf{\Omega}}$. The cross section, $\Sigma_{t}$, defines the probability that the particles are removed through both absorption and scattering, (i.e. $\Sigma_{t}=\Sigma_{a}+\Sigma_{s}$ ) with the source term designated as $S_{g}$. For criticality problems, the eigenvalue form of the BTE reads as,

$$
\begin{array}{r}
\hat{\boldsymbol{\Omega}} \cdot \nabla \psi_{g}(\boldsymbol{r}, \hat{\boldsymbol{\Omega}})+\Sigma_{t, g}(\boldsymbol{r}) \psi_{g}(\boldsymbol{r}, \hat{\boldsymbol{\Omega}})=\int_{4 \pi} \sum_{g^{\prime}=1}^{G} \Sigma_{s, g^{\prime} \rightarrow g}\left(\boldsymbol{r}, \hat{\boldsymbol{\Omega}}^{\prime}, \rightarrow \hat{\boldsymbol{\Omega}}\right) \psi_{g^{\prime}}\left(\boldsymbol{r}, \hat{\boldsymbol{\Omega}}^{\prime}\right) \mathrm{d} \hat{\Omega}^{\prime}+ \\
\lambda \frac{\chi_{g}}{4 \pi} \sum_{g^{\prime}=1}^{G} \nu \Sigma_{f, g^{\prime}}(\boldsymbol{r}) \int_{4 \pi} \psi_{g^{\prime}}\left(\boldsymbol{r}, \hat{\boldsymbol{\Omega}}^{\prime}\right) \mathrm{d} \hat{\Omega}^{\prime}, \\
\forall g \in\{1,2,3 \ldots, G\},
\end{array}
$$

where the fission energy spectrum is denoted as $\chi_{g}, \nu_{g}$ is the average number of neutrons emitted per fission and $\Sigma_{f, g}$ the fission cross-section. We consider both the angular flux $\psi_{g}$ and eigenvalue $\lambda=1 / k_{\text {eff }}$. In the following sections it is sufficient to consider just the fixed source mono-energetic equations.

Both vacuum and reflective boundary conditions will be considered in this article which are defined as,

$$
\psi(\boldsymbol{r}, \hat{\mathbf{\Omega}})=0, \quad \text { where } \quad \hat{\boldsymbol{\Omega}} \cdot \boldsymbol{n}<0 .
$$

and,

$$
\hat{\boldsymbol{\Omega}} \cdot \boldsymbol{n}=-\hat{\boldsymbol{\Omega}}^{\prime} \cdot \boldsymbol{n} \quad \text { and } \quad\left(\hat{\boldsymbol{\Omega}}^{\prime} \times \hat{\boldsymbol{\Omega}}\right) \cdot \boldsymbol{n}=0,
$$

respectively. The term $\boldsymbol{n}$ denotes the outward facing normal to the boundary and $\hat{\boldsymbol{\Omega}}^{\prime}$ is the specular reflected angle to $\hat{\boldsymbol{\Omega}}$ with respect to $\boldsymbol{n}$. Satisfying these conditions using the wavelet discretisation is discussed in section 3 .

The angular dimension $\hat{\boldsymbol{\Omega}}$ is conventionally represented on the surface of the sphere in terms of its azimuthal $\omega$ and polar $\theta$ directions as shown in figure 1. The unit vector components describing the projection of $\hat{\Omega}$ in Cartesian space can alternatively be denoted as $\hat{\boldsymbol{\Omega}}=$ $\left(\hat{\Omega}_{x}, \hat{\Omega}_{y}, \hat{\Omega}_{z}\right)=\left(\left(1-\mu^{2}\right)^{\frac{1}{2}} \cos \omega,\left(1-\mu^{2}\right)^{\frac{1}{2}} \sin \omega, \mu\right)$, where $\mu=\cos \theta$. 


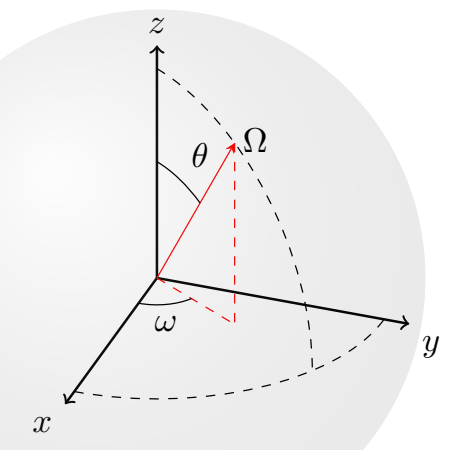

Figure 1: Particle travel on unit sphere, $\hat{\mathbf{\Omega}}$ denoting the direction of particle travel. The azimuthal direction is denoted by $\omega$, and the polar direction by $\theta$.

\subsection{The General Angular Discretisation}

The angular flux $\psi(\boldsymbol{r}, \hat{\boldsymbol{\Omega}})$ is approximated using an arbitrary scheme is given by,

$$
\psi(\boldsymbol{r}, \hat{\mathbf{\Omega}}) \approx \sum_{j=1}^{\mathcal{M}} \psi_{j}(\boldsymbol{r}) \mathcal{G}_{j}(\hat{\mathbf{\Omega}}),
$$

where $\mathcal{G}_{j}(\hat{\boldsymbol{\Omega}})$ represent the expansion's angular basis functions and $\psi_{j}(\boldsymbol{r})$ are the respective expansion coefficients. The angular discretised equations are formed by substituting the approximation into equation 1 , multiplying the equation by each basis function $\mathcal{G}_{i}$, for $i \in$ $\{1,2, \ldots, G\}$, and integrating over the angular domain,

$$
\begin{array}{r}
\int \mathcal{G}_{i}(\hat{\boldsymbol{\Omega}})\left\{\hat{\boldsymbol{\Omega}} \cdot \nabla+\Sigma_{t}(\boldsymbol{r})\right\} \sum_{j=1}^{\mathcal{M}} \psi_{j}(\boldsymbol{r}) \mathcal{G}_{j}(\hat{\boldsymbol{\Omega}}) \mathrm{d} \boldsymbol{\Omega}- \\
\int \mathcal{G}_{i}(\hat{\boldsymbol{\Omega}})\left\{\int_{4 \pi} \Sigma_{s}\left(\boldsymbol{r}, \hat{\boldsymbol{\Omega}}^{\prime} \rightarrow \hat{\boldsymbol{\Omega}}\right) \sum_{j=1}^{\mathcal{M}} \psi_{j}(\boldsymbol{r}) \mathcal{G}_{j}\left(\hat{\boldsymbol{\Omega}}^{\prime}\right) \mathrm{d} \boldsymbol{\Omega}^{\prime}\right\} \mathrm{d} \boldsymbol{\Omega}=\int \mathcal{G}_{i}(\hat{\boldsymbol{\Omega}}) S(\boldsymbol{r}, \hat{\boldsymbol{\Omega}}) \mathrm{d} \boldsymbol{\Omega} \\
\forall i \in\{1,2,3 \ldots, \mathcal{M}\} .
\end{array}
$$

This can be written as the following matrix system which is the generalised angular discretised form of the BTE,

$$
\boldsymbol{A}_{\boldsymbol{x}} \frac{\partial}{\partial x} \boldsymbol{\Psi}(\boldsymbol{r})+\boldsymbol{A}_{\boldsymbol{y}} \frac{\partial}{\partial y} \boldsymbol{\Psi}(\boldsymbol{r})+\boldsymbol{A}_{\boldsymbol{z}} \frac{\partial}{\partial z} \boldsymbol{\Psi}(\boldsymbol{r})+\boldsymbol{H} \boldsymbol{\Psi}(\boldsymbol{r})=\boldsymbol{S}(\boldsymbol{r}) .
$$


The terms $\boldsymbol{A}_{\boldsymbol{x}}, \boldsymbol{A}_{\boldsymbol{y}}$ and $\boldsymbol{A}_{\boldsymbol{z}}$ take the form of $\mathcal{M} \times \mathcal{M}$ angular discretised streaming matrices. $\boldsymbol{H}$ is the $\mathcal{M} \times \mathcal{M}$ matrix containing the discretised scattering and removal operators and $\boldsymbol{S}(\boldsymbol{r})$ is an $\mathcal{M}$-sized vector containing the discretised external source term. Lastly, the $\mathbf{\Psi}(\boldsymbol{r})$ term is a vector of size $\mathcal{M}$ containing the expansion coefficients of the angular flux at every spatial position. Derivation of all matrices and source vectors for an arbitrary set of angular basis functions $G_{i}$ in the expansion of equation 5 can be found in [6].

\subsection{The Subgrid Scale Finite Element Spatial Discretisation}

This section presents the SGS discretisation of the angular discretised BTE's spatial variable $\boldsymbol{r}$ [34]. The scheme is based on a finite element formulation for which the problem domain $V$ is partitioned $(\mathcal{T})$ into a mesh of a finite set of non overlapping elements. The SGS formulation uses a multiscale representation of the full solution $\Psi(\boldsymbol{r})$ that is decomposed into two components $\boldsymbol{\Psi}(\boldsymbol{r})=\boldsymbol{\Phi}(\boldsymbol{r})+\boldsymbol{\Theta}(\boldsymbol{r})$, where $\boldsymbol{\Phi}(\boldsymbol{r})$ and $\Theta(r)$ represent the coarse scale and fine scale components of the solution respectively.

The coarse component's approximation lies in a continuous finite element space, spanned by the continuous basis functions $N_{j}, j \in\left\{1,2, \ldots, n_{N}\right\}$, associated with the FEM mesh. The fine approximation lies in a discontinuous space, spanned by the discontinuous basis functions $Q_{j}, j \in\left\{1,2, \ldots, n_{Q}\right\}$, also associated with the FEM mesh. In this work both the continuous and discontinuous FEM functions are piecewise linear across the elements of the mesh. The flux vector $\boldsymbol{\Psi}(\boldsymbol{r})$ is then expressed as

$$
\boldsymbol{\Psi}(\boldsymbol{r}) \approx \widetilde{\boldsymbol{\Phi}}(\boldsymbol{r})+\widetilde{\boldsymbol{\Theta}}(\boldsymbol{r})=\sum_{j}^{n_{N}} \boldsymbol{N}_{j}(\boldsymbol{r}) \boldsymbol{\Phi}_{j}+\sum_{j}^{n_{Q}} \boldsymbol{Q}_{j}(\boldsymbol{r}) \boldsymbol{\Theta}_{j}
$$

where $\boldsymbol{\Phi}_{j}$ and $\boldsymbol{\Theta}_{j}$ are angular vectors of size $\mathcal{M}$ containing the respective expansion coefficients, and the terms $\boldsymbol{N}_{j}$ and $\boldsymbol{Q}_{j}$ are $\mathcal{M} \times \mathcal{M}$ diagonal matrices containing the FEM basis function at the node $j$. Equation 7 is re-written in terms of its residual as: $\mathcal{R}_{e}(\boldsymbol{\Psi})=(\boldsymbol{A} \cdot \nabla+\boldsymbol{H}(\boldsymbol{r})) \boldsymbol{\Psi}(\boldsymbol{r})-$ $\boldsymbol{S}(\boldsymbol{r})=0$. This is then weighted using both sets of functions $\boldsymbol{N}_{i}, i \in\left\{1,2, \ldots, n_{N}\right\}$, and $\boldsymbol{Q}_{i}, i \in\left\{1,2, \ldots, n_{Q}\right\}$, and integrated over the spatial domain. A system of $n_{N}+n_{Q}$ equations is formed, which is defined by

$$
\begin{aligned}
\int_{V} \boldsymbol{N}_{i} \mathcal{R}_{e}(\widetilde{\boldsymbol{\Phi}}) \mathrm{d} V+\int_{V} \boldsymbol{N}_{i} \mathcal{R}_{e}(\widetilde{\boldsymbol{\Theta}}) \mathrm{d} V=0, & \forall j \in\left\{1,2, \ldots, n_{N}\right\} \\
\int_{V} \boldsymbol{Q}_{i} \mathcal{R}_{e}(\widetilde{\boldsymbol{\Phi}}) \mathrm{d} V+\int_{V} \boldsymbol{Q}_{i} \mathcal{R}_{e}(\widetilde{\boldsymbol{\Theta}}) \mathrm{d} V=0, & \forall j \in\left\{1,2, \ldots, n_{Q}\right\} .
\end{aligned}
$$


where the integral subscript $V$ denotes integration over volume. The discretised system of equations can be written as

$$
\left[\begin{array}{ll}
A & B \\
C & D
\end{array}\right]\left[\begin{array}{c}
\widetilde{\boldsymbol{\Phi}} \\
\widetilde{\boldsymbol{\Theta}}
\end{array}\right]=\left[\begin{array}{l}
\widetilde{\boldsymbol{S}}_{\Phi} \\
\widetilde{\boldsymbol{S}}_{\Theta}
\end{array}\right],
$$

where $\widetilde{\boldsymbol{\Phi}}$ and $\widetilde{\boldsymbol{\Theta}}$ are vectors of size $n_{N} \mathcal{M}$ and $n_{Q} \mathcal{M}$, respectively, containing the angular coefficient on the nodes of the coarse/fine (respectively) finite element mesh. The matrices and vectors are defined in equations 11 through 16.

$$
\begin{gathered}
A_{i, j}=\int_{V} \boldsymbol{N}_{i} \boldsymbol{H} \boldsymbol{N}_{j} \mathrm{~d} V-\int_{V} \nabla \boldsymbol{N}_{i} \cdot \boldsymbol{A} \boldsymbol{N}_{j} \mathrm{~d} V+\int_{\Gamma} \boldsymbol{N}_{i}(\boldsymbol{A} \cdot \boldsymbol{n}) \boldsymbol{N}_{j} \mathrm{~d} \Gamma \\
B_{i, j}=\int_{V} \boldsymbol{N}_{i} \boldsymbol{H} \boldsymbol{Q}_{j} \mathrm{~d} V-\int_{V} \nabla \boldsymbol{N}_{i} \cdot \boldsymbol{A} \boldsymbol{Q}_{j} \mathrm{~d} V \\
C_{i, j}=\sum_{V_{e} \in \mathcal{T}} \int_{V_{e}} \boldsymbol{Q}_{i} \boldsymbol{H} \boldsymbol{N}_{j} \mathrm{~d} V+\sum_{V_{e} \in \mathcal{T}} \int_{V_{e}} \boldsymbol{Q}_{i} \boldsymbol{A} \cdot \nabla \boldsymbol{N}_{j} \mathrm{~d} V \\
D_{i, j}=\sum_{V_{e} \in \mathcal{T}} \int_{V_{e}} \boldsymbol{Q}_{i} \boldsymbol{H} \boldsymbol{Q}_{j} \mathrm{~d} V-\sum_{V_{e} \in \mathcal{T}} \int_{V_{e}} \nabla \boldsymbol{Q}_{i} \cdot \boldsymbol{A} \boldsymbol{Q}_{j} \mathrm{~d} V+\sum_{V_{e} \in \mathcal{T}} \int_{\Gamma_{\text {out }}^{e}} \boldsymbol{Q}_{i} \boldsymbol{R}_{r}^{\text {out }} \boldsymbol{Q}_{j} \mathrm{~d} \Gamma \\
\widetilde{\boldsymbol{S}}_{\Phi_{i}}=\sum_{j} \int_{V} \boldsymbol{N}_{i} \boldsymbol{N}_{j} \boldsymbol{S}_{j} \mathrm{~d} V \\
\widetilde{\boldsymbol{S}}_{\Theta_{i}}=\sum_{j} \int_{V} \boldsymbol{Q}_{i} \boldsymbol{Q}_{j} \boldsymbol{S}_{j} \mathrm{~d} V .
\end{gathered}
$$

The integration subscripts $V_{e}$ and $\Gamma$ denote integration over the element's $(e)$ volume and the problem's surface, respectively. The integral with subscript $\Gamma_{\text {out }}$ denotes an integration across the problem surface involving the outward flowing particles, this is discussed more fully in the following paragraphs. Also the term $\boldsymbol{A}$ that has been introduced denotes the vector of matrices $\left(A_{x}, A_{y}, A_{z}\right)$. Each matrix is of size $\mathcal{M} \times \mathcal{M}$ and forms the $i, j^{\text {th }}$ block component (of size $\mathcal{M} \times \mathcal{M})$ of the matrices in equation 10. Similarly each vector is of size $\mathcal{M}$ and forms the $i^{\text {th }}$ block vector (of size $\mathcal{M}$ ) of the vectors in equation 10 . The surface integral in equations 11 and 14 results from the application of Green's Theorem upon the advection operator. It 
contains both incoming and outgoing information through the boundary, but this can be decomposed into separate components through the use of Riemann methods [18, 34, 35]. The details are covered in [34] but, for any arbitrary angular discretisation, the surface integral (e.g. in equation 11) can be written in terms of incoming and outgoing components,

$$
\int_{\Gamma} \boldsymbol{N}_{i}(\boldsymbol{A} . \boldsymbol{n}) \boldsymbol{N}_{j} \mathrm{~d} \Gamma \boldsymbol{\Phi}_{j}=\int_{\Gamma_{\text {in }}} \boldsymbol{N}_{i} \boldsymbol{R}_{r}^{i n} \boldsymbol{N}_{j} \mathrm{~d} \Gamma \boldsymbol{\Phi}_{j}+\int_{\Gamma_{\text {out }}} \boldsymbol{N}_{i} \boldsymbol{R}_{r}^{\text {out }} \boldsymbol{N}_{j} \mathrm{~d} \Gamma \boldsymbol{\Phi}_{j} .
$$

The Riemann matrices $\boldsymbol{R}_{r}^{i n}$ and $\boldsymbol{R}_{r}^{\text {out }}$ are formed and operate on the flux vector $\boldsymbol{\Phi}_{j}$ in such a way that information flows only in or out of the element through its surface. Resolving boundary conditions is therefore relatively straight forward, for example void conditions are resolved by simply zeroing out the incoming integral. However a thorough review on how to derive the linear systems of each component, along with resolving general boundary conditions can be found in [34]. A key component of the SGS formulation is that the fine scale incoming information is assumed to be zero. This has already been applied to equation 14 where the Riemann method has been applied to the surface integral and only the outgoing integral is retained. This decouples the discontinuous variables from those belonging to other elements. It means that the fine scale component acts locally to refine the solution within each element.

An expression for the fine (discontinuous) solution $\tilde{\boldsymbol{\Theta}}$ can be formed by multiplying the lower part of the linear system in equation 10 by $\boldsymbol{D}^{-1}$,

$$
\widetilde{\boldsymbol{\Theta}}=-D^{-1} C \widetilde{\boldsymbol{\Phi}}+D^{-1} \widetilde{\boldsymbol{S}}_{\Theta} .
$$

This can be replaced into the upper part of equation 10 to reformulate the equations in terms of the coarse variables $\tilde{\boldsymbol{\Phi}}$,

$$
\left(A-B D^{-1} C\right) \widetilde{\boldsymbol{\Phi}}=\widetilde{\boldsymbol{S}}_{\Phi}-B D^{-1} \widetilde{\boldsymbol{S}}_{\Theta}
$$

As shown in [34], the advantage of solving equation 19 comes from the fact that only the coarse scale variables are solved for, which are typically much smaller in size than the fine scale variables. However solving equation 19 is dependent on the inversion of the matrix $D$ which is, in fact, the largest of all matrices involved. It was shown in [34] that $D^{-1}$ can be computed efficiently due to the manipulation of the boundary integrals involving the discontinuous variables - see equation 14. This results in the discontinuous components being coupled only to those on a common element, which means the matrix $D$ is block structured, and so its inversion could be performed on each individual sub-matrix separately. Each block matrix is of size $n_{Q} N_{e} \times n_{Q} N_{e}$, where $N_{e}$ denotes the number of nodes on the element. This inversion of the individual blocks of $D$ makes the whole inversion significantly more efficient. However when the angular expansion sizes become large the inversion of $D$ can become computationally expensive. A solution to this problem is presented in the next section. 


\subsection{The "Efficient" SGS Discretisation}

As mentioned in section 2.3, the matrix $D$ is element block-diagonal, making it simple to invert $D$. In this article, we refer to any discretisation scheme shown that uses this form of $D$ as "classical". Therefore, for a given mesh of $\mathcal{E}$ elements with $\mathcal{N}$ nodes on each element, the computation and storage of this "classical" form of $D^{-1}$ requires the inversion and storage of $\mathcal{E}$ sub-matrices of size $\mathcal{N} \mathcal{M} \times \mathcal{N} \mathcal{M}$ (where $\mathcal{M}$ is the number of angles on the node). Provided the size of these sub-matrices is relatively small their computation and storage is cheap. However, whilst the number of nodes on a typical individual element is relatively small $(\mathcal{N}<10)$ the size of the angular expansion is often large $(\mathcal{M}>>100)$.

For large numbers of angles, it becomes prohibitively expensive to invert "classical" $D$. Given this, below we introduce an "efficient" SGS discretisation that uses a form of $D$ with greater sparsity. The "efficient" approach presented here is based on the method detailed in Buchan and Pain [35], who applied this to the discretised $P_{n}$ equations. Buchan and Pain [35] showed that certain entries of the sub-matrices could be set to zero (i.e., making $D$ more sparse) without adverse effects on the solution; all that is required from $D^{-1}$ is "sufficient" stability in the discretisation.

The exact form of this "efficient" approach to inverting $D$ is dependent on the angular discretisation, but generally involves finding further block-diagonal structure that provides sufficient stability within the element blocks of $D$. If we denote each element block of $D$ as $D^{e}$, with structure

$$
D^{e}=\left(\begin{array}{ccc}
D_{1,1}^{e} & D_{1,2}^{e} & \ldots D_{1, \mathcal{M}}^{e} \\
D_{2,1}^{e} & D_{2,2}^{e} & \ldots D_{2, \mathcal{M}}^{e} \\
\vdots & \vdots & \ddots \\
D_{\mathcal{M}, 1}^{e} & D_{\mathcal{M}, 2}^{e} & \ldots D_{\mathcal{M}, \mathcal{M}}^{e}
\end{array}\right)
$$

where each sub-matrix $D_{i, j}^{e}$ is the $\mathcal{N} \times \mathcal{N}$ matrix containing the spatially discretised information relating to angular moments $i, j$. Directly inverting equation 20 is the "classical" scheme described above. In this work, we form the "efficient" scheme by ensuring the angular moments couple only between themselves (this is different to the approach taken for $P_{n}$ shown in Buchan and Pain [35]); all the off-diagonal block matrices of equation 20 are set to zero, namely

$$
D^{e}=\left(\begin{array}{ccc}
D_{1,1}^{e} & 0 & \ldots 0 \\
0 & 0 & \ldots 0 \\
\vdots & \vdots & \ddots \\
0 & 0 & \ldots D_{\mathcal{M}, \mathcal{M}}^{e}
\end{array}\right)
$$


With this structure the inversion can be performed on the individual $\mathcal{N} \times \mathcal{N}$ blocks $\mathrm{D}_{i, i}^{e}, i \in$ $\{1,2, \ldots \mathcal{M}\}$. As the inversion of a general matrix of dimension $d$ is of complexity $O\left(d^{3}\right)$, the costs of the inversion of equation 21 is $O\left(\mathcal{M N}^{3}\right)$, as opposed to equation 20 whose inversion has complexity $O\left(\mathcal{M}^{3} \mathcal{N}^{3}\right)$. Storage costs of the matrix $\mathrm{D}^{e}$ are now $O\left(\mathcal{M N}^{2}\right)$ as opposed to $O\left(\mathcal{M}^{2} \mathcal{N}^{2}\right)$. The fact that both complexity and storage is linear with $\mathcal{M}$ means that for large angle sizes the cost of inversion and storage of $D$ is manageable.

Despite the zeroing of elements of $D$, the solutions to both hyperbolic and diffusive problems remain stable and accurate, as demonstrated in section 4. Most importantly there are also no adverse effects regarding the conservation of particles, and the solution will converge to the true solution as the mesh resolution is increased. A discussion of these properties is contained in [35], which shows global conservation of particles is maintained for an arbitrary angular discretisation scheme. In fact it is demonstrated in the article that the $D$ matrix could be manipulated in any way, and the effects were only on the formulation's ability to stabilise the solution. This article is using these findings to manipulate the $D$ matrix as already described.

\section{The Haar Wavelet Formulation for the Angular Discretisation of the BTE}

\subsection{The Multiresolution Analysis}

A multiresolution analysis (MRA) defined on 1 dimensional domains comprises of a nested sequence of functional subspaces $V_{j}$ of the function space $L^{2}(\mathcal{R})$, where $\mathcal{R}$ is defined here as a subset of the real line [6]. The space $L^{2}(\mathcal{R})$ is defined as the set of functions $f$ that satisfy the condition $\int_{\mathcal{R}} f^{2} \mathrm{~d} \mathcal{R}<\infty$. The nested sequence of closed subspaces $V_{j}, j \in\{0,1,2, \ldots, \infty\}$ must satisfy the following conditions:

1. $V_{j} \subset V_{j+1}, \quad \forall j \in\{0,1,2, \ldots, \infty\}$;

2. The union of all closed subspaces is dense in the functional subspace $L^{2}(\mathcal{R})$, i.e. $\bigcup_{j=1}^{\infty} V_{j}=L^{2}(\mathcal{R})$;

3. For every level $j$, there exists a set of scaling functions $\phi_{j, n}, n \in N^{\prime}(j)$ for some index set $N^{\prime}$, forming a Riesz basis of $V_{j}$.

In numerical applications, the nested spaces of the MRA can be used in approximating a function $f$ onto the $L^{2}(\mathcal{R})$ functional space. This is accomplished by projecting $f$ onto $f_{j} \in$ 
$V_{j}$, for $j=\{0,1,2, \ldots, \infty\}$. Since the scaling functions $\phi_{j, n}$ span $V_{j}$, the projected function $f_{j}$ can be written as an expansion of the scaling functions, after which the approximation takes the form

$$
f \rightarrow f_{j}=\sum_{n} \alpha_{j, n} \phi_{j, n}
$$

where $\alpha_{j, n}$ are the expansion coefficients. Of note is that the accuracy of the approximation is determined by the selection of $j$ as condition 2 posits the full recovery of the solution as $j \rightarrow \infty$.

\subsection{The General Wavelet MRA}

A general wavelet basis can be defined as $\psi_{j, b} \mid b \in B(j)$ for some index set $B(j)$ where $j$ denotes the wavelet level, and $b$ indexes a wavelet belonging to the level. These wavelets form a basis for the $W_{j}$ space which have the property of complementing the $V_{j}$ space in the $V_{j+1}$, such that $V_{j+1}=V_{j} \oplus W_{j}$ for all $j=\{0,1,2, \ldots, \infty\}$. Since $W_{j}$ is contained in the $V_{j+1}$ space, this implies that each wavelet function $\psi_{j, b}$ must be able to be constructed from a combination of the scaling function spanning the $V_{j+1}$ space. That is,

$$
\psi_{j, b}=\sum_{n \in N^{\prime}(j+1)} h_{j, b, n} \phi_{j+1, n} .
$$

Using a recursive relationship and applying condition 1 , the space $V_{j}$ can be written in terms of $V_{0}$ and $W_{n}$ for $n=0$ to $j-1$ as

$$
V_{j}=V_{j-1} \oplus W_{j-1}=V_{j-2} \oplus W_{j-2} \oplus W_{j-1}=\ldots=V_{0} \oplus_{n=0}^{j-1} W_{n} .
$$

Combining equation 24 with condition 2 , it is possible to decompose the $L^{2}(\mathcal{R})$ space into the $V_{o}$ and $W_{j}$ space for $j=\{0,1,2, \ldots, \infty\}$ :

$$
L^{2}(\mathcal{R})=V_{0} \oplus_{j=0}^{\infty} W_{j}
$$

This essentially defines a new basis of the $L^{2}(\mathcal{R})$ space using the wavelets contained in $W_{j}$ and the scaling function contained in $V_{0}$. As such, any function that is an element of the $L^{2}(\mathcal{R})$ space can also be written in terms of the wavelet functions spanning the $W_{j}$ and $V_{0}$ spaces as

$$
f=\sum_{n \in N^{\prime}(0)} \alpha_{0, n} \phi_{0, n}+\sum_{l=0}^{\infty} \sum_{n \in B(l)} \beta_{l, n} \psi_{l, n} .
$$


In a similar manner, projecting $f$ onto $f_{j} \in V_{j}$ yields

$$
f_{j}=\sum_{n \in N^{\prime}(0)} \alpha_{0, n} \phi_{0, n}+\sum_{l=0}^{j-1} \sum_{n \in B(l)} \beta_{l, n} \psi_{l, n} .
$$

\subsection{The Haar Wavelet MRA defined on the 2D Surface of the Sphere}

For 1 dimensional Haar wavelets, the space $V_{j}$ for $j=\{0,1,2, \ldots, \infty\}$ is defined over the interval $[-1,1]$ that is subdivided into $2^{j}$ equal sized intervals, as shown in figure 2 . The spaces $V_{j}$ contain the set of functions that are piecewise constant over these intervals. The scaling

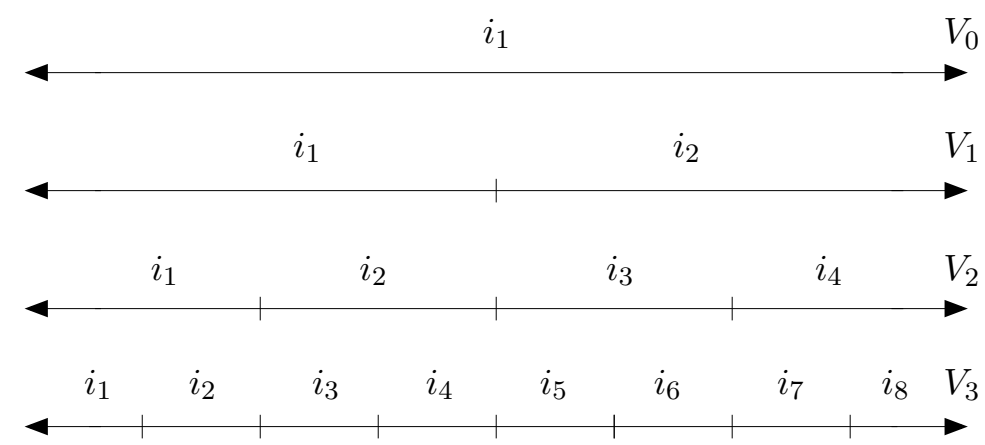

Figure 2: Partitioning of the interval [-1,1] associated with the Haar wavelet MRA spaces $V_{j}$.

functions chosen to span the space $V_{j}$ are simply the control volume-like functions that are each associated with a partitioned interval, over which they have the value 1 and are zero elsewhere. That is, the scaling function associated with $n^{\text {th }}$ interval, denoted here as $i_{n}^{j}$, of the space $V_{j}$ is defined by,

$$
\phi_{j, n}(x)= \begin{cases}1, & \text { if } x \in i_{n}^{j}, \\ 0, & \text { otherwise }\end{cases}
$$

The Haar wavelets that form the basis for the space $W_{j}$ are defined over the partitioning defined by the space $V_{j+1}$. The size of the basis is $2^{j}$, and each function is again associated with an interval $i_{n}^{j}$, and is defined by,

$$
\psi_{j, n}(x)= \begin{cases}1, & \text { if } x \in i_{2 n-1}^{j+1} \\ -1, & \text { if } x \in i_{2 n}^{j+1} \\ 0, & \text { otherwise }\end{cases}
$$


for $n \in\left\{1,2, \ldots, 2^{j}\right\}$. These functions define the wavelets and scaling functions in the general expansion of equation 27 , where the lowest order scaling functions are those associated with the space $V_{0}$. To simplify notation the expansion 27 is arranged into a single expansion of the form,

$$
f(x)=\sum_{i=1}^{2^{j}} h_{i} \gamma_{i}(x)
$$

where $h_{i}$ represents the expansion coefficients, and $\gamma_{i}(x)$ the scaling and wavelet functions. Whilst the expansion defined here is on the interval $[-1,1]$, extending this to arbitrary intervals is trivial through the suitable scaling and translating of these basis functions. An illustration of the way in which the piecewise constant functions are expanded on the wavelet space is shown in figure 3.

Extending this approximation to 2 dimensional planes, one applies a tensor product of two expansions in the form of equation 30 that resolve the separate dimensions, say $\mu, \omega$. This is given as,

$$
\psi(\mu, \omega)=\sum_{i_{1}}^{2^{j_{1}}} h_{i_{1}}^{\mu} \gamma_{i_{1}}(\mu) \sum_{i_{2}}^{2^{j_{2}}} h_{i_{2}}^{\omega} \gamma_{i_{2}}(\omega)=\sum_{i}^{2^{j_{1}} 2^{j_{2}}} h_{i} \gamma_{i}(\mu, \omega),
$$

which has again been combined into a single summation involving 2 dimensional Haar functions, $\gamma_{i}(\mu, \omega)$. These functions are defined from the products of two functions from the two separate 1D Haar expansions, i.e. $\gamma_{i}(\mu, \omega)=\gamma_{i_{1}}(\mu) \gamma_{i_{2}}(\omega)$, for $i_{1} \in\left\{1,2, \ldots 2^{j_{1}}\right\}$ and $i_{2} \in\left\{1,2, \ldots 2^{j_{2}}\right\}$. This expansion is used to form the approximation over the surface of the sphere defined by the azimuthal and polar plane $(\omega, \mu)$.

In this article eight separate 2D wavelet expansions in the form of equation 31 are used to resolve the full angular domain, one to resolve each of the 8 octants defining the sphere's surface, this is shown in figure 4a. Note that the resolution in the azimuthal and polar dimensions are free to vary independently, and may be set to provide suitable resolution across the two dimensions. The lowest order approximation $\left(j_{1}=j_{2}=0\right)$ is piecewise constant across the eight octants as shown in figure $4 \mathrm{c}$, this is equivalent to $S_{2}$ in figure 4a. Figure $4 \mathrm{~d}$ presents the partitioning of the approximation $\left(j_{1}=j_{2}=2\right)$ showing each octant being divided into regular $4 \times 4$ grids. As a comparison the equal weighted Kershaw \& Harte [36] $S_{6}$ discretisation is presented in figure $4 \mathrm{~b}$. The centre of the patches and their area define the directions and weights of the quadrature schemes respectively.

The wavelet discretisation will always divide up the surface of the sphere into regular shaped patches in the same fashion as figures $4 \mathrm{c}$ and $4 \mathrm{~d}$, where the discretisation will depend on the 


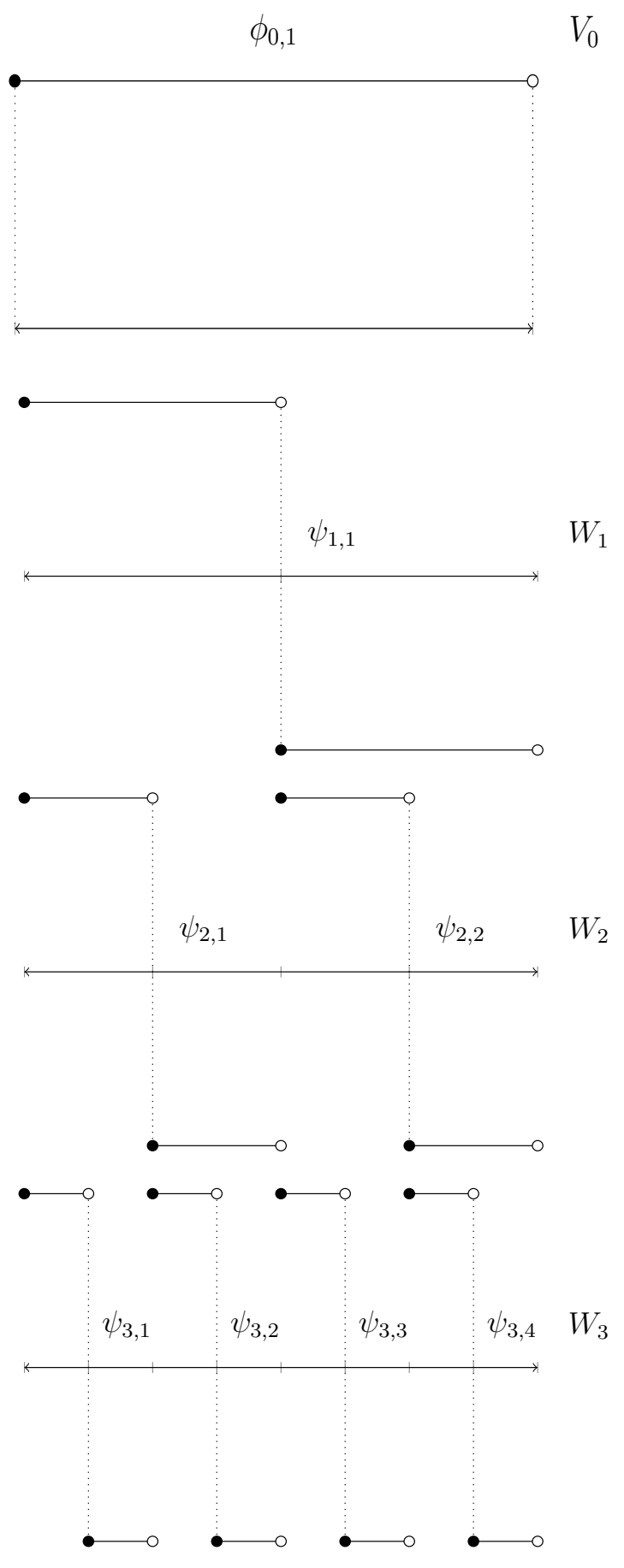

Figure 3: Partitioning of the interval $[-1,1]$ associated with the Haar wavelet MRA spaces $V_{j}$. 
resolution set for each octant and for each angular dimension. The wavelet approximation will also be identical to that when the control volume scaling functions are used on the same sphere's surface partitioning. That is, consider a set of $\mathcal{M}$ patches and an associated set of $\mathcal{M}$ control volume functions, each associated with a patch, which have the value 1 over their patch and 0 elsewhere. Using these control volume functions and performing the angular integrals, to get to equation 7 , results in the $S_{n}$ method with equal weights and directions defined as the centre of each patch. Therefore there is an equivalent relationship between $S_{n}$ and Haar wavelet discretisation in that they result in the same solution when the $S_{n}$ quadrature set is defined in this manner.

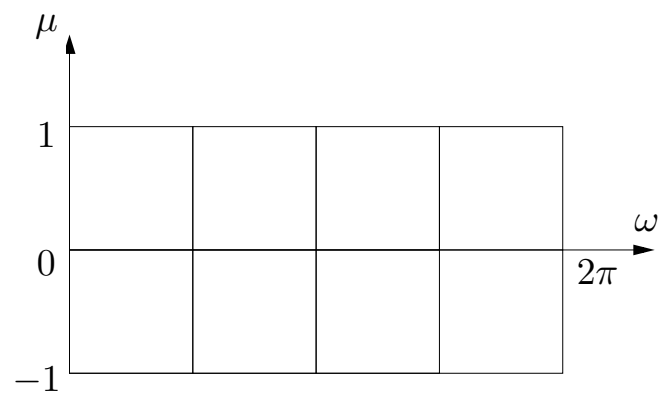

(a) $S_{2}$

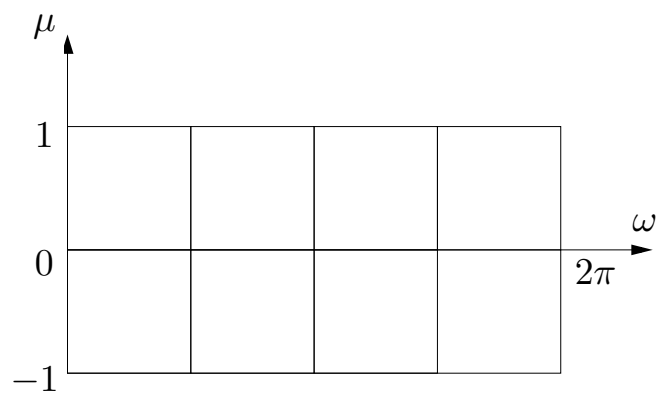

(c) $W_{1,1}$

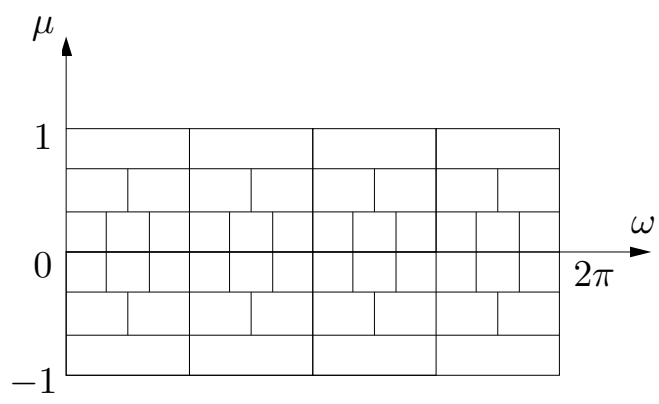

(b) $S_{6}$

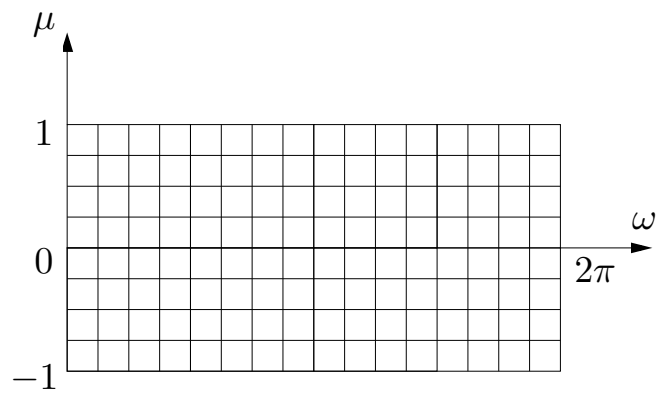

(d) $W_{3,3}$

Figure 4: Quadrature schemes of the Haar Wavelet and equal weighted $S_{n}$ discretisations. The sphere is partitioned into patches over which both schemes provide approximations with piece-wise constant variation. The centre of each patch can also be viewed as defining the directions of the $S_{n}$ method and their area being the weight. 


\section{Numerical Examples}

Two numerical examples are presented in this section to demonstrate the use of Haar wavelets in resolving radiation transport problems. In the first example, a radiation shielding problem is presented, with the second example a benchmark multigroup criticality eigenvalue problem. All problems tested are two-dimensional and the resulting linear systems are solved using the (matrix-free spatial) multigrid preconditioned GMRES algorithm outlined in [37].

Section 2.4 described two different approaches for forming $D$; a "classical" and an "efficient" method. To distinguish these appraoches the classical and efficient Haar wavelet solutions are denoted by $C W_{n, m}$ and $E W_{n, m}$, respectively, where $n$ denotes the polar expansion order and $m$ the azimuthal expansion order. As the numerical examples are both 2D problems, only the 4 octants in the upper hemisphere $(\mu \geq 0)$ are required to be resolved, and for simplicity we enforce the same resolution to all octants. The size of the $2 \mathrm{D}$ Haar wavelet angular discretisation is $2^{n+m}$ at each spatial point.

The focus of these numerical examples centre on two themes. The first is to demonstrate that the treatment of the spatial discretisation as detailed in section 2.4 does not result in a reduction of accuracy. That is the method can substantially increase computational efficiency without incurring errors. The second is to demonstrate the Haar wavelets have the potential to adapt in angle by their elimination from the approximation when their expansion coefficient is smaller than a defined tolerance level. This will set the focus of the second article that enables the self-adaptation (or self-selection) of the wavelet functions.

In the analysis presented comparisons are drawn against the solutions obtained from the $S_{n}$ method where the equal weighted Kershaw \& Harte [36] quadrature scheme is used. To ensure consistency the same SGS discretisation is employed, and a GMRES solver with multi-grid based preconditioners are used. It should be noted that due to these solvers the $S_{n}$ will not be optimal in terms of computational solving times, i.e. these are not sweep based solvers.

\subsection{Dog-Leg Duct in 2D}

The first example is a two-dimensional, mono-energetic shielding problem containing a source adjacent to a dog-leg shaped duct; a schematic is shown in figure 5a. Region I contains a source with an intensity of 1 neutron $\mathrm{cm}^{-3} \mathrm{~s}^{-1}$, with region II a weak scattering duct and 
region III a heavy absorbing material. The cross sections for each region are listed in table 1. Vacuum boundary conditions are employed on all four edges of the geometry. Three nodes numbered 1-3, and located at coordinate points $(1.5,1.5),(6,10.5)$, and $(7.5,16)$ respectively are also defined on the problem domain in figure $5 \mathrm{a}$.

A reference solution is shown in figure $5 \mathrm{c}$, using an $S_{40}$ equal weighted angular discretisation. The $S_{40}$ discretisation uses more than twice the number of angular degrees of freedom compared to the largest $C W_{n, m}$ or $E W_{n, m}$ wavelet formulation presented in this example; thus, its solution is considered a good reference for an error measure. The $S_{40}$ solution shows a diffuse-like flux spectrum with a peak scalar flux value of approximately 1.34 neutrons $\mathrm{cm}^{-2} \mathrm{~s}^{-1}$ in the source region. This peak is heavily attenuated through the duct, with the scalar flux roughly 5 orders of magnitude smaller at the end of the duct. This calculation was carried out on the unstructured spatial mesh shown in figure 5b with 13058 elements.

\begin{tabular}{cccc}
\hline \hline Region & Source $\left(\mathrm{cm}^{-3} \mathrm{~s}^{-1}\right)$ & $\Sigma_{a}\left(\mathrm{~cm}^{-1}\right)$ & $\Sigma_{s}\left(\mathrm{~cm}^{-1}\right)$ \\
\hline I & 1.0 & 0.5 & 0.0 \\
II & 0.0 & 0.0 & 0.005 \\
III & 0.0 & 0.5 & 0.0 \\
\hline \hline
\end{tabular}

Table 1: The region cross-sections for the dog-leg problem shown in figure 5a.

For most of the results shown in this section, we examine the angular convergence relative to the aforementioned reference solution $\psi_{\text {ref }}$, computed on the mesh shown in figure $5 \mathrm{~b}$ with $S_{40}$ angular discretisation. We compute the $\mathrm{L}^{2}$ error norm, $\epsilon$, of the scalar flux of a solution $\psi$ given by

$$
\epsilon=\left[\int_{V}\left(\psi^{\text {scalar }}-\psi_{\text {ref }}^{\text {scalar }}\right)^{2} \mathrm{~d} V\right]^{\frac{1}{2}},
$$

where the scalar superscript denotes the scalar neutron flux, attained by integrating the angular flux at each node over the entire angular domain of $4 \pi$.

\subsubsection{Spatial convergence}

We begin by investigating the spatial convergence of the Haar wavelet angular discretisation. In particular, we examine whether the "efficient" SGS discretisation described in section 2.4 affects the convergence rate. For this test, we compare the classical formulation, $C W_{n, m}$, and the efficient, $E W_{n, m}$, with a fixed wavelet order of $n=m=3$. The reference solution used 


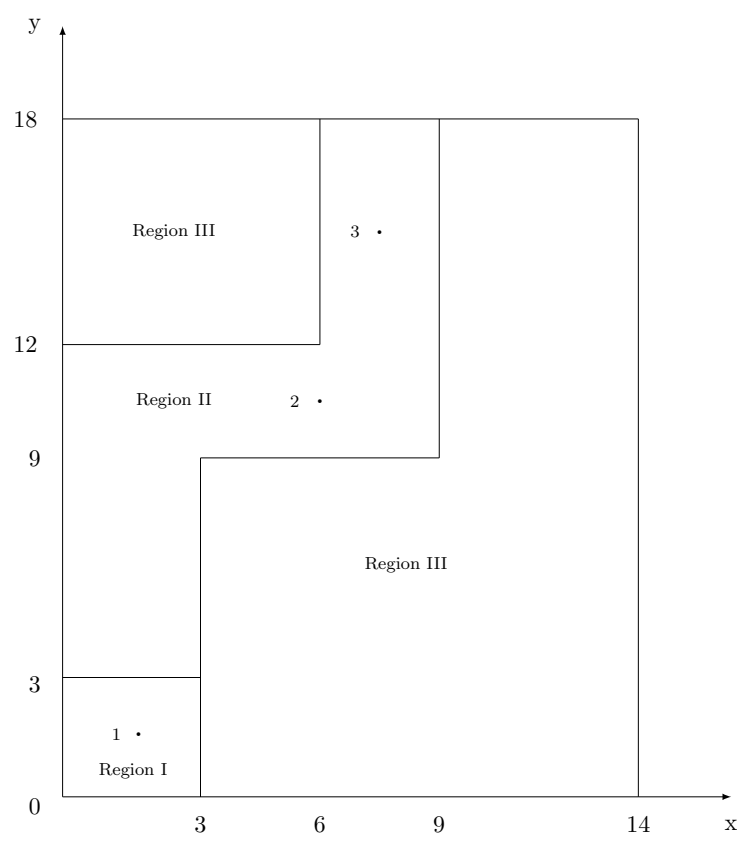

(a) A schematic of the geometry for this dog-leg duct (dimensions in $\mathrm{cm}$ ). Three nodes numbered 1-3 are referenced in figures below and are located at coordinate points $(1.5,1.5),(6,10.5)$, and $(7.5,16)$.
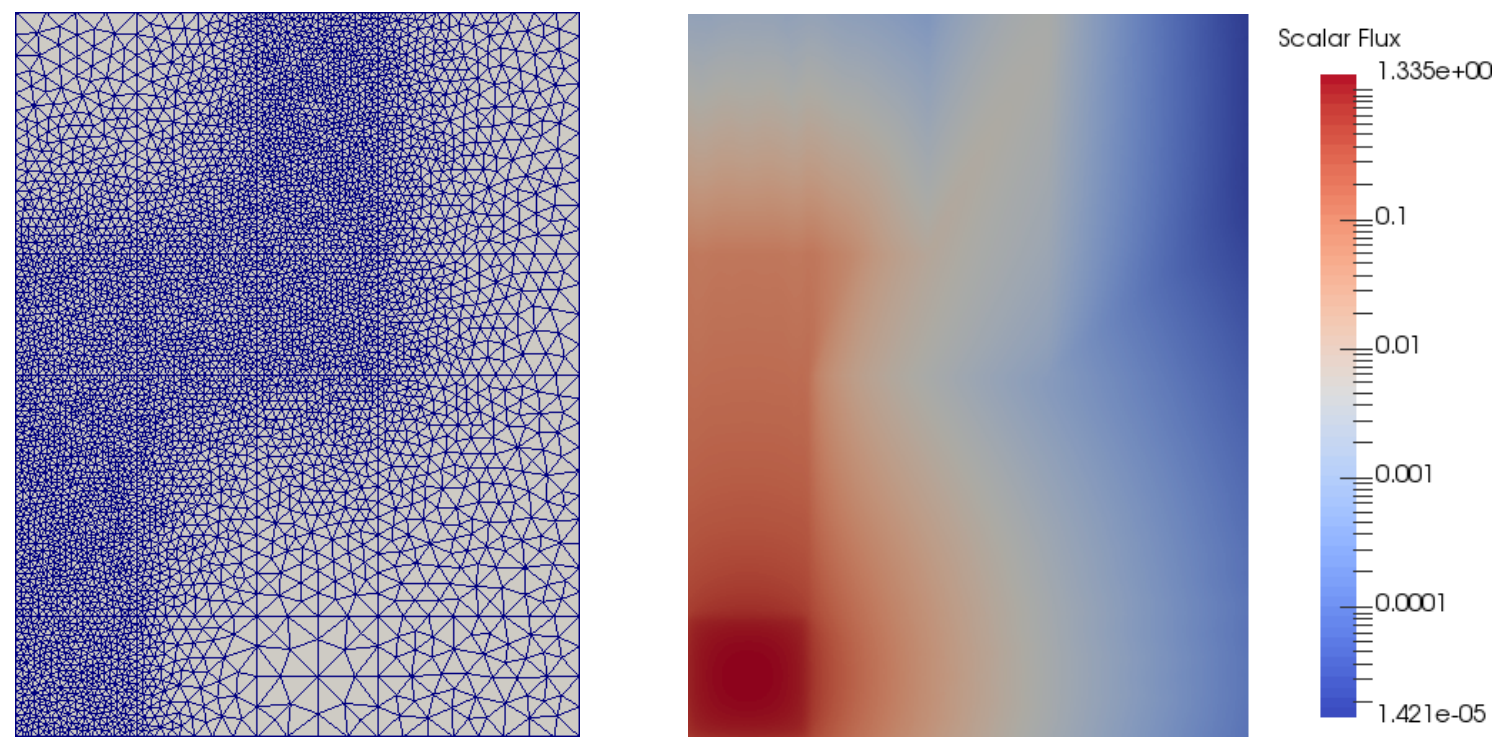

(b) Unstructured reference spatial mesh with 13058 (c) The scalar flux across the domain with $S_{40}$ elements, used to discretise the domain shown in angular discretisation.

figure 5 a.

Figure 5: Description of the dog-leg problem outlined in section 4.1. 
was computed using a $C W_{n, m}$ formulation with $n=m=3$ and a spatial mesh containing 167374 elements.

Five spatial meshes with 8727, 13058, 24388, 32025 and 61042 elements were used to illustrate the spatial convergence in this section (labeled mesh 1 through 5, respectively, in figure 6). Figure 6 plots the squared error in the scalar flux across the line $(0,6)-(14,6)$ for these meshes. The line $(0,6)$ - $(14,6)$ was chosen as it represented an area in the domain where the error was most significant.

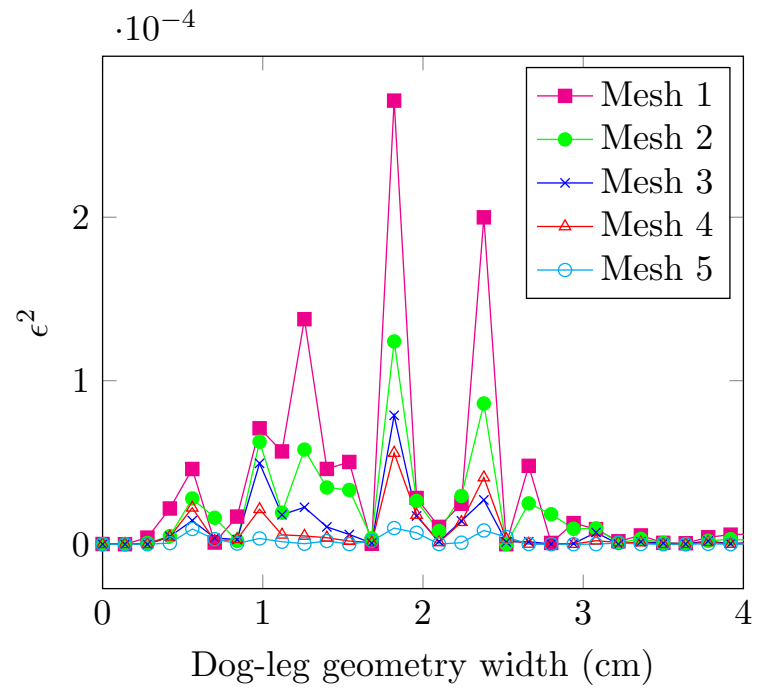

(a) $E W_{3,3}$

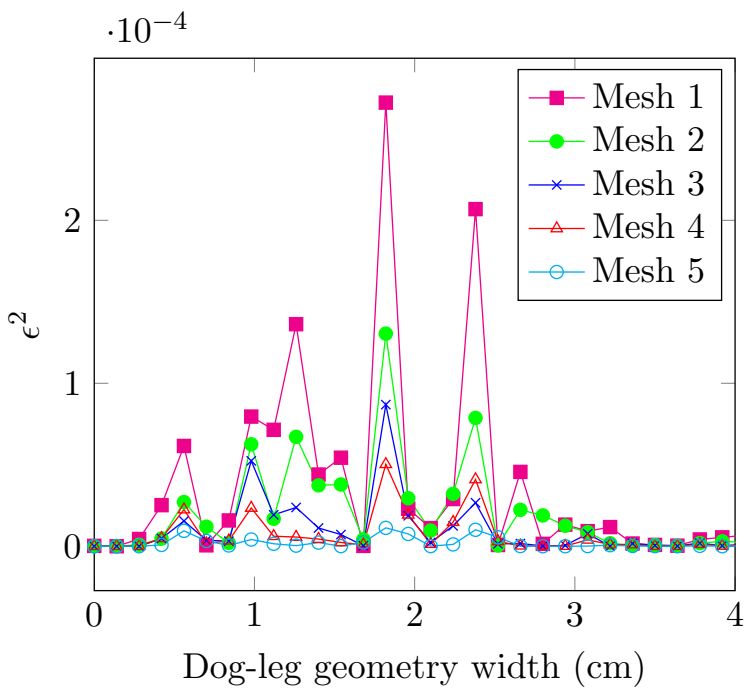

(b) $C W_{3,3}$

Figure 6: The squared error in the scalar flux using the (a) $E W_{n, m}$ and (b) $C W_{n, m}$ Haar wavelet formulations when solving the dog-leg problem. Five meshes with increasing resolution $(8727,13058,24388,32025$ and 61042 elements, labelled mesh 1 through 5, respectively) were employed and the errors were measured along the line $(0,6)-(14,6)$. In order to focus on the pertinent regions of the problem domain with significant error, this plot only shows the scalar flux squared error up to $4 \mathrm{~cm}$ of the geometry width. The scalar flux squared error drops to zero beyond $4 \mathrm{~cm}$. Raw scalar flux error values had positive and negative biases, hence the squared error was plotted instead so to enable analysis on a consistent scale.

Figure 6 shows the error to be most pronounced on the coarse spatial meshes for both the $C W_{n, m}$ and $E W_{n, m}$ formulations, as expected. The largest errors are also approximately located in the same regions on the mesh. In fact, the $E W_{n, m}$ formulation shown in figure 6 a appears to introduce very little additional error to the solution when compared with the $C W_{n, m}$ formulation shown in figure $6 \mathrm{~b}$. The error reduces significantly in both formulations with further mesh refinement. This is illustrated further in figure 7 , where the $\mathrm{L}^{2}$ error across the domain, $\epsilon$, is plotted against the refined meshes. This result shows that the $E W_{n, m}$ and 
$C W_{n, m}$ formulations achieve almost identical accuracy, and that the convergence rate of the discretisation schemes is the same.

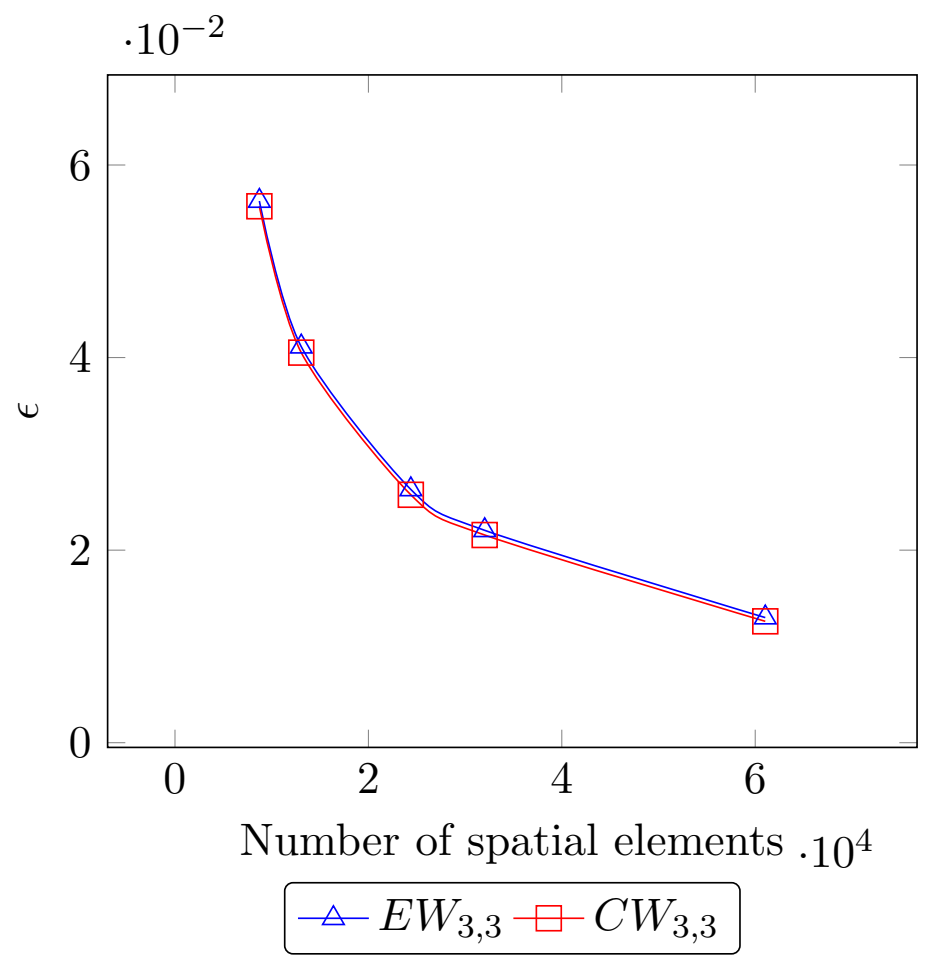

Figure 7: The spatial convergence of the $\epsilon$ using both the $E W_{n, m}$ and $C W_{n, m}$ Haar wavelet formulations for the dog-leg problem. The five data points in the plot correspond to meshes with 8727, 13058, 24388, 32025 and 61042 respective spatial elements.

\subsubsection{Angular convergence}

We now turn to examining the accuracy of the Haar wavelet discretisation and the convergence as the number of angles is increased. First, we compare the $C W_{n, m}$ and $E W_{n, m}, n=$ $m=\{1,2,3,4\}$ Haar wavelet formulations against $S_{n}$ orders with roughly equivalent angular expansion sizes - the spatial mesh used is Mesh 2 which has 13058 elements. This is shown in figure 8, where we have increased the azimuthal and polar orders equally. Figure 8a shows the error against the number of angles present in the angular discretisation. Similar to section 4.1.1, we see that the "efficient" wavelet formulation achieves almost identical accuracy when compared to the classical. We also see that our wavelet formulation produces roughly comparable error to the $S_{n}$ method as the number of angles is increased, which is expected, 
as both methods are first order. The difference in errors comes from the difference in the quadrature of the two-dimensional sphere, for the two discretisations (this will be discussed further below).

Figures $4 \mathrm{a}$ and $4 \mathrm{c}$ show that $S_{2}$ and $W_{1,1}$ are equivalent and we see this reflected in the identical errors shown figure $8 \mathrm{a}$. As the order is increased, we can see in figures $4 \mathrm{~b}$ and $4 \mathrm{~d}$ that the quadratures are no longer equivalent. When mapped to the sphere, the $W_{3,3}$ discretisation shown in figure $4 \mathrm{~d}$ will place more angles close to the poles of the sphere than a (roughly) equivalent equal weighted $S_{n}$ discretisation (again see figure 4b). Furthermore, in problems with two spatial dimensions, our wavelet formulation will "line-up" angles with constant $\omega$, exacerbating ray-effects (this effect disappears in three-dimensions due to non-overlap of the $\omega$ angle). These two differences are responsible for the differences in errors seen in figure $8 \mathrm{a}$ and as mentioned, will be examined further below.

Importantly, figure 8b shows that though the $C W_{n, m}$ and $E W_{n, m}$ produce the same error, the solve time has been reduced by almost 2 orders of magnitude for the highest wavelet order with the "efficient" formulation. This is due to the inversion of the $D^{-1}$ matrix detailed in section 2.4, which is the major cost given large angle size. In particular, we see the runtime of the efficient scheme growing linearly with angle size, while the classical scheme is growing super-linearly. Given the identical accuracy and improved solve time, we now focus exclusively on the efficient wavelet scheme.

Given the difference in quadratures between an equal weighted $S_{n}$ scheme and our wavelet formulation, and the subsequent difference in errors seen in figure 8a, it seems natural to examine the effect of using different polar and azimuthal orders with our wavelets. Indeed this is one benefit to using our wavelet scheme; it is very easy to preference polar or azimuthal resolution if we know a priori that it would be beneficial. Figure 9 presents convergence results where the polar and azimuthal orders were varied independently. For simplicity, we chose to fix the polar resolution, $n=\{1,2,3,4\}$ and then increase the azimuthal order, while again comparing against $S_{n}$ orders with roughly equivalent angular expansion sizes. This is partially driven by a desire to (roughly) match the equal weighted $S_{n}$ quadrature.

Figure 9a shows the error given the number of angles present in each wavelet expansion. We can see that wavelet order $E W_{1, m}, m=\{1,2, \ldots, 7\}$ begins with an identical error as $S_{2}$ and then converges faster than $S_{n}$ until we reach around 50 angles. However, since the polar resolution was fixed, the $E W_{1, m}$ reaches a point where further refinement in the azimuthal does not help increase the accuracy of the solution. As such, to further increase the accuracy 


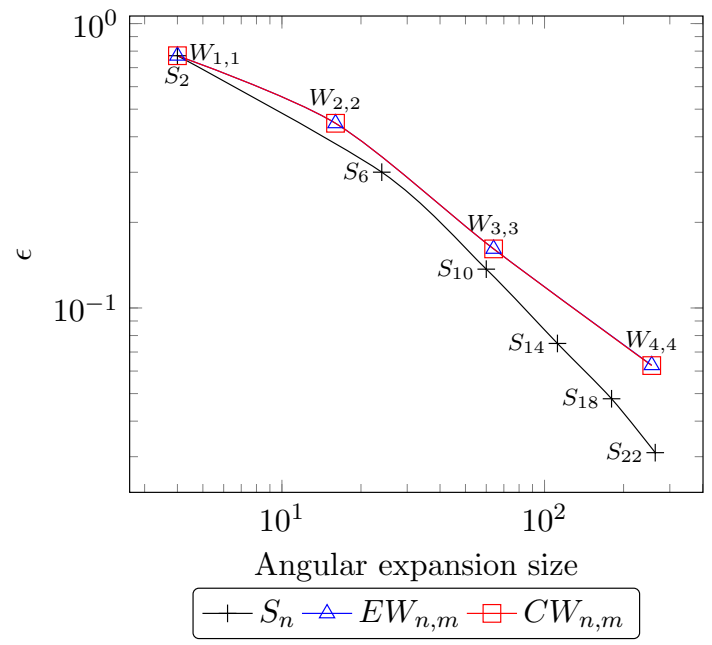

(a) Error vs the number of angles

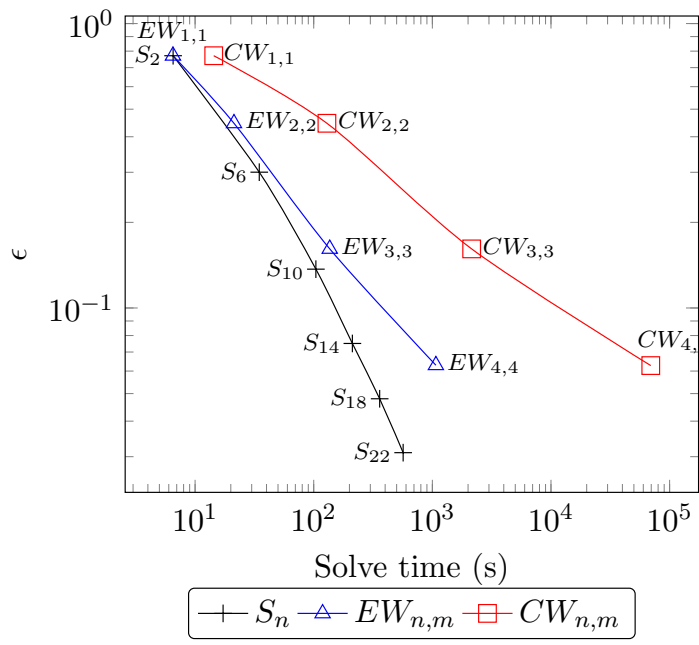

(b) Error vs solve time

Figure 8: Comparisons of the convergence of the $C W_{n, m}$ and $E W_{n, m}(n=m=\{1,2,3,4\})$ schemes, compared to $S_{n}, n=\{2,6, \ldots, 22\}$. Each data point in the plot uses a spatial mesh with 13058 elements.

of the solution, we must increase the resolution in the polar direction. Contrasting this, is the $E W_{4, m}, m=\{1,2, \ldots, 4\}$ expansion, which starts with a similar error as $S_{2}$ and converges similarly, though it has significantly higher error at higher orders given the same number of angles. This is due to the the over-resolution in the polar direction.

Obviously we must balance the polar and azimuthal resolution in a given problem to achieve the greatest accuracy, though figure 9a suggests that increasing the order unevenly while preferencing the azimuthal, say from $W_{1,1}, W_{1,2}, W_{2,3}, W_{2,4}$ to $W_{3,5}$, would achieve very similar results to an equal weighted $S_{n}$ scheme. The convergence results shown in figure 9 a are reflected in the solve time shown in figure $9 \mathrm{~b}$, because as figure $8 \mathrm{~b}$ shows, our solve time for the "efficient" wavelet scheme is linearly related to the number of angles present (regardless of where those angles are placed on the sphere). The $W_{3,4}$ and $W_{3,5}$ expansions for example, produce roughly the same error as $S_{14}$ and $S_{22}$, respectively, while also matching the solve time.

Given we can produce roughly equivalent solve times and errors with our wavelet formulation when compared to $S_{n}$, a natural question to ask is why bother with wavelets? Regardless of any other differences, the angular matrices in a wavelet formulation will always be significantly less sparse than an equivalent $S_{n}$ quadrature (which are diagonal), increasing the amount of work required to perform common operations like matrix-vector products. Thankfully, 


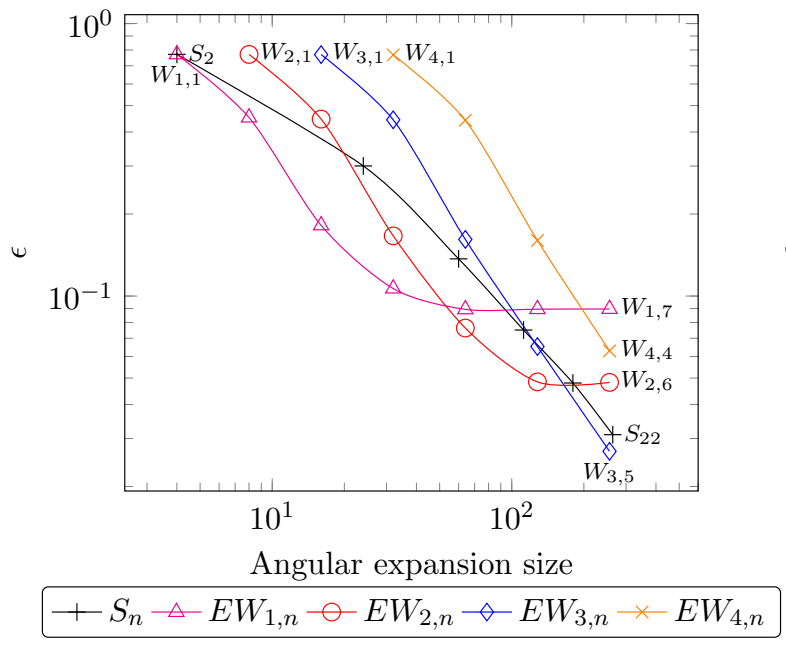

(a) Error vs the number of angles

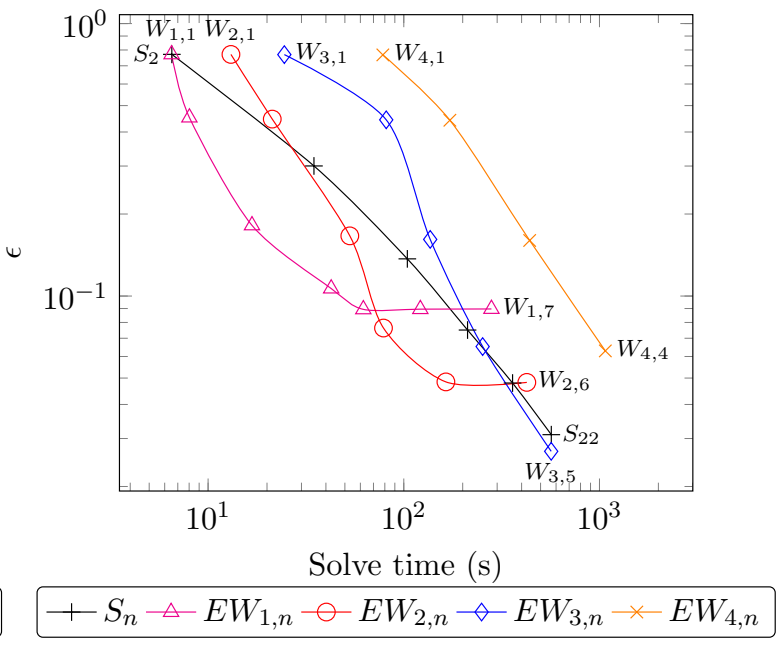

(b) Error vs solve time (s)

Figure 9: Comparisons of the convergence of the "efficient" wavelet scheme, $E W_{n, m}$, for various orders: $E W_{1, m}$, $m=\{1,2, \ldots, 7\}, E W_{2, m}, m=\{1,2, \ldots, 6\}, E W_{3, m}, m=\{1,2, \ldots, 5\}$, and $E W_{4, m}, m=\{1,2, \ldots, 4\}$, compared to $S_{n}, n=\{2,6, \ldots, 22\}$. Each data point in the plot uses a spatial mesh with 13058 elements.

the use of a Haar wavelet expansion allows angular adaptivity to be used easily, as the importance of a wavelet can be tied to the magnitude of its angular coefficient. Large angular coefficients contribute significantly to the solution whereas small coefficients add little. Therefore, discarding wavelets with small angular coefficients will not affect the solution to a large degree, but will reduce the size of the expansion required to accurately represent the angular flux. This can be performed on a node-by-node basis, allowing the ability to adapt the angular resolution easily across space (and subsequently remove the need to pick an "optimal" quadrature for each problem as above).

Figure 10 shows a grouping of the angular flux coefficients of a Haar wavelet expansion $\left(E W_{4,4}\right)$ according to the size of their coefficients. Generally, we can see that a large number of the wavelet coefficients are very small and therefore contribute little to the solution. In this problem, the coefficients with large angular coefficients correspond to wavelets "pointing" down streaming paths (and other areas of importance). Defining an arbitrary threshold tolerance value, $\tau$, many wavelet functions below the threshold could be excluded from the expansion set, resulting in a reduction in computational time. It is expected that the accuracy of the solution will not be severely compromised with the exclusion of these minimally contributing wavelet functions. 
Table 2 shows the percentage reduction in total angle size when thresholding has been applied to the basis functions below two arbitrary tolerances (see [38] for a discussion on thresholding tolerances). We can see that excluding all angular wavelet coefficients below the value of $10^{-3}$ results in a $6.25 \%$ reduction in number of angles present inside the source region at node 1. This number increased to $64.84 \%$ for node 2 (inside the duct but further away from the source region). In this problem, table 2 shows a particular trend, namely that the further away one is from the source, the less the number of angles are required to obtain a precise solution within a defined tolerance value. Consequently, closer to the source region, the reduction in computation size is much less. This is due to the significant streaming paths found far from the source (i.e., in the duct).

\begin{tabular}{cccc}
\hline \hline Node & Tolerance $\tau$ & No. Angles & Reduction no. angles(\%) \\
\hline 1 & \multirow{2}{*}{$10^{-3}$} & \multirow{2}{*}{256} & 6.3 \\
2 & & & 64.8 \\
3 & & & 63.3 \\
\hline 1 & \multirow{2}{*}{$10^{-4}$} & 256 & 0.0 \\
2 & & & 52.7 \\
3 & & 54.7 \\
\hline \hline
\end{tabular}

Table 2: The percentage reduction in number of angles after thresholding has been applied, for two different thresholding tolerances, $\tau$, at the three nodes shown in figure $5 \mathrm{a}$. 


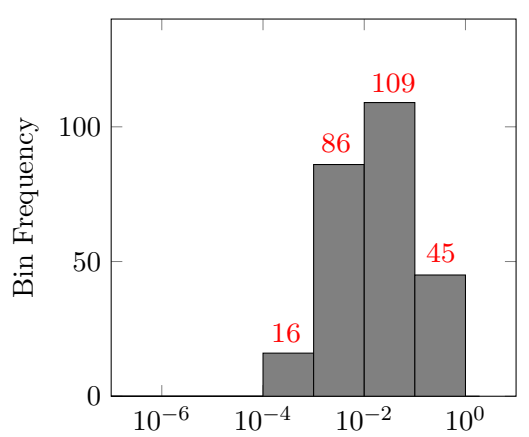

Magnitude of angular flux coefficients

(a) Node 1

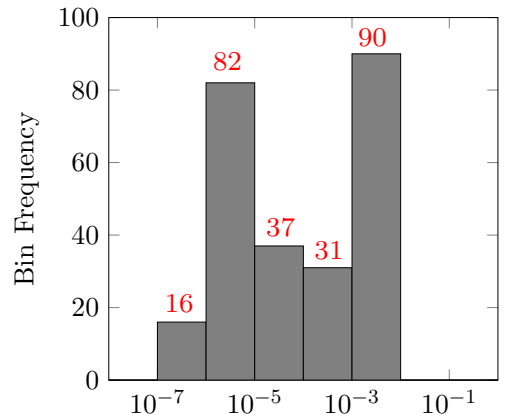

Magnitude of angular flux coefficients

(b) Node 2

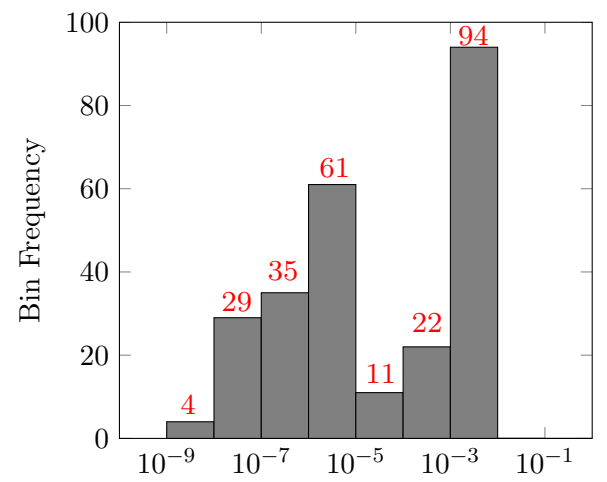

Magnitude of angular flux coefficients

(c) Node 3

Figure 10: The distribution of angular flux coefficients for the $E W_{4,4}$ solution at the predefined nodes on the dog-leg mesh. The $E W_{4,4}$ solution uses 256 angular expansions. The number above each histogram bin represents the number of angular coefficients that fall within range of that bin. Each bin is an order of magnitude in width. The sum of all bins equals 256 . 


\subsection{The C5 MOX Fuel Assembly}

The second example is a two-dimensional, seven group criticality eigenvalue benchmark problem proposed by the OECD/NEA. Specifications of the quarter mini-core MOX fuel assembly are outlined by Cavarec et al. [39]. The energy ranges of the material nuclear cross-section data are presented in figure 11a. The quarter core, presented in figure 11b, has a square geometry with reflective boundary conditions applied to its left and bottom side, and vacuum boundary conditions to its top and right side. It is comprised of four fuel assemblies, each with a dimension of $21.42 \times 21.42 \mathrm{~cm}$, while the overall dimension of the quarter core is $64.26 \times 64.26 \mathrm{~cm}$. Two of the assemblies use $\mathrm{UO}_{2}$ fuel, while the remaining two employ a mixture of $\mathrm{UO}_{2}$ fuel and MOX fuel. Each assembly houses a $17 \times 17$ array of pin cells. The pin cell configuration is shown in figure 11c, where region I corresponds to a homogenised fuel-clad mix, and region II to the moderator material. The number densities for the fuel, cladding and moderator region, as well as group scatter cross-sections, and neutron production for each group are specified in the work of Cathalau et al. [40]. The simulations were run on an unstructured spatial mesh with 40148 elements. Two nodes located respectively at coordinate points $(10,10)$, and $(50,50)$ are also defined. To avoid excessive clutter, these are not explicitly labelled in figure $11 \mathrm{~b}$.

The profiles for the fast and thermal neutron scalar flux solutions, using the $E W_{4,4}$ discretisation, are presented in figure 12. These show large variations in the flux profiles, where the fast flux (group 1) peaks over the central region of the core, whilst the thermal flux (group 7) peaks across those regions containing a moderator. In figure $13 \mathrm{a}$ and $13 \mathrm{~b}$ the respective results for the $k_{\text {eff }}$ with an $S_{n}$ and $E W_{n, m}$ discrestisation are compared to the MCNP results of Cavarec

et al. [39]. For small angular expansions each discretisation has a different error, however, as the expansion order is increased both $S_{n}$ and $E W_{n, m}$ converge to the benchmark solution.

Line plots of the scalar flux across the problem domain comparing the $E W_{4,4}$ and $S_{22}$ methods for neutron groups 1 and 7 are also examined. The scalar flux is computed along the line $(0,0)-(64.26,64.26)$ of the problem domain. The results are shown in figure 14 and again show good agreement between the $S_{n}$ and $E W_{n, m}$ discretisations. 


\begin{tabular}{cc}
\hline \hline Group & Upper Energy \\
1 & $20 \mathrm{MeV}$ \\
2 & $1 \mathrm{MeV}$ \\
3 & $500 \mathrm{keV}$ \\
4 & $3 \mathrm{eV}$ \\
5 & $0.625 \mathrm{eV}$ \\
6 & $0.1 \mathrm{keV}$ \\
7 & $0.02 \mathrm{keV}$ \\
\hline \hline
\end{tabular}

(a) The C5 MOX fuel assembly neutron group cut-off energies.

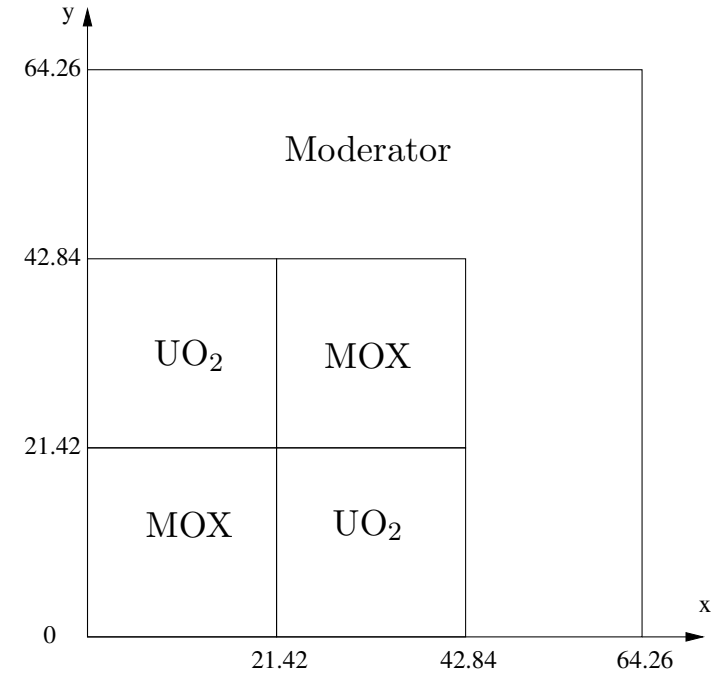

(b) The C5 MOX fuel assembly configuration and geometry with dimensions in centimetres.

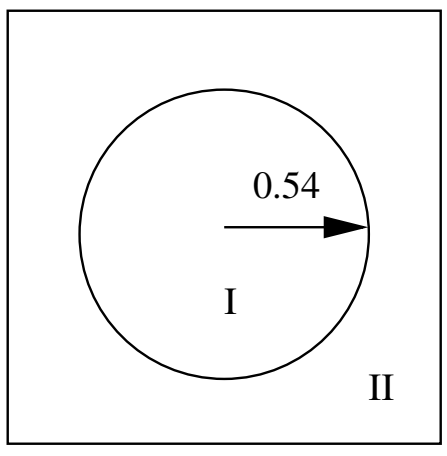

1.26

(c) The C5 MOX fuel assembly pincell geometry with dimensions in centimetres.

Figure 11: The C5 MOX fuel assembly pin cell, core configuration and geometry. 

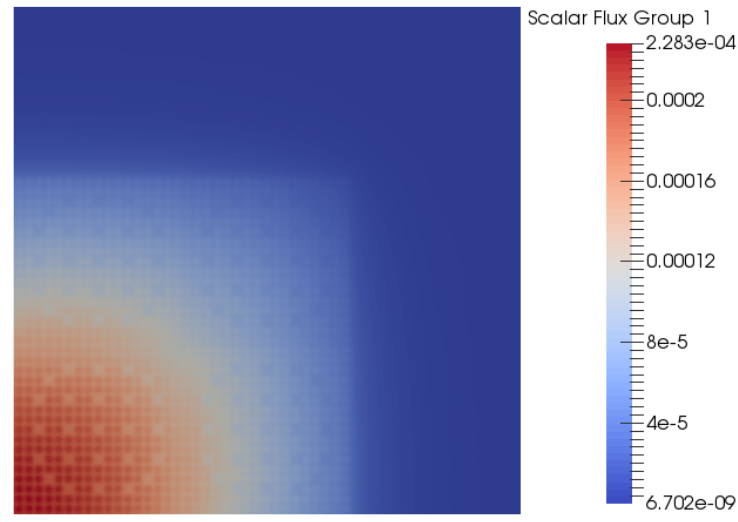

(a) Group 1 scalar flux

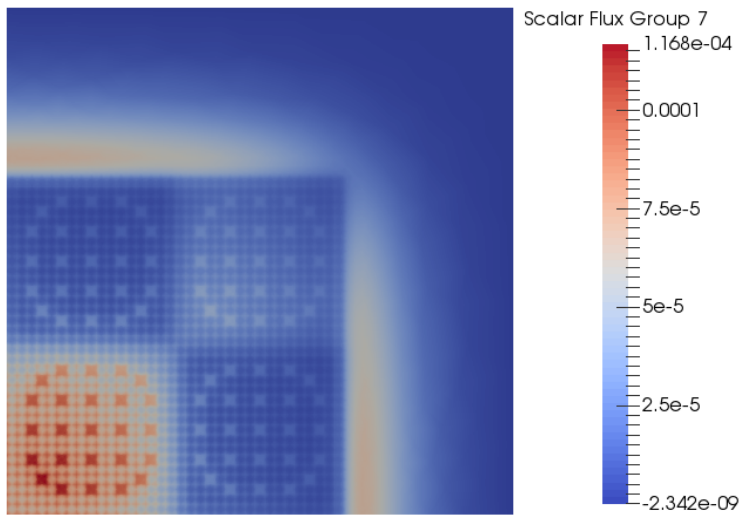

(b) Group 7 scalar flux

Figure 12: The C5 MOX assembly $E W_{4,4}$ scalar flux solutions for neutron groups 1 and 7 .
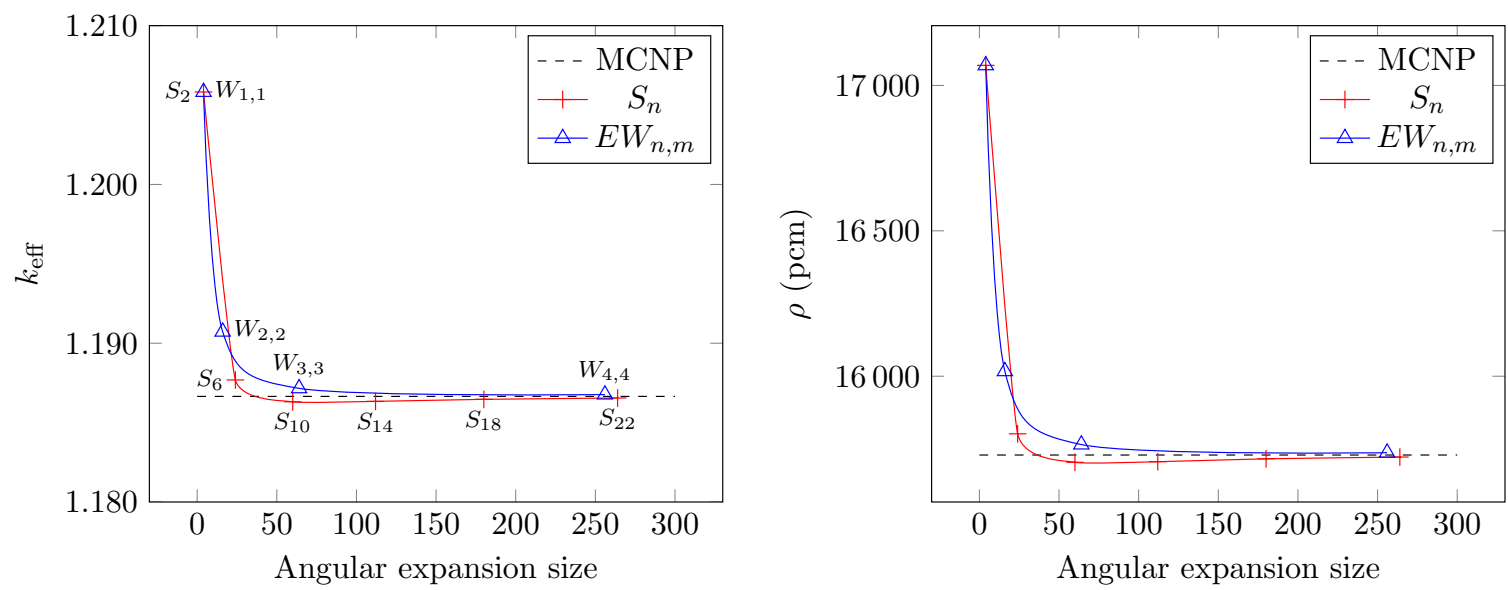

(a) $k_{\text {eff }}$ for the different discretisation schemes. (b) $\rho$ in pcm for the different discretisation schemes, MCNP percent error listed in [39] is 0.008 . where $\rho=\frac{k_{\text {eff }}-1}{k_{\text {eff }}} \times 10^{5}$.

Figure 13: The C5 MOX fuel assembly reactivity for the different discretisation schemes. 


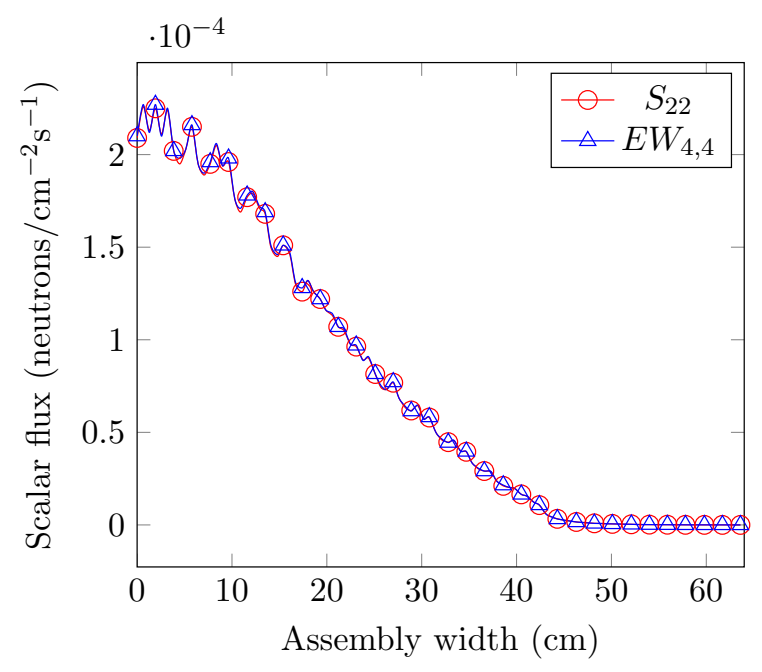

(a) Neutron group 1

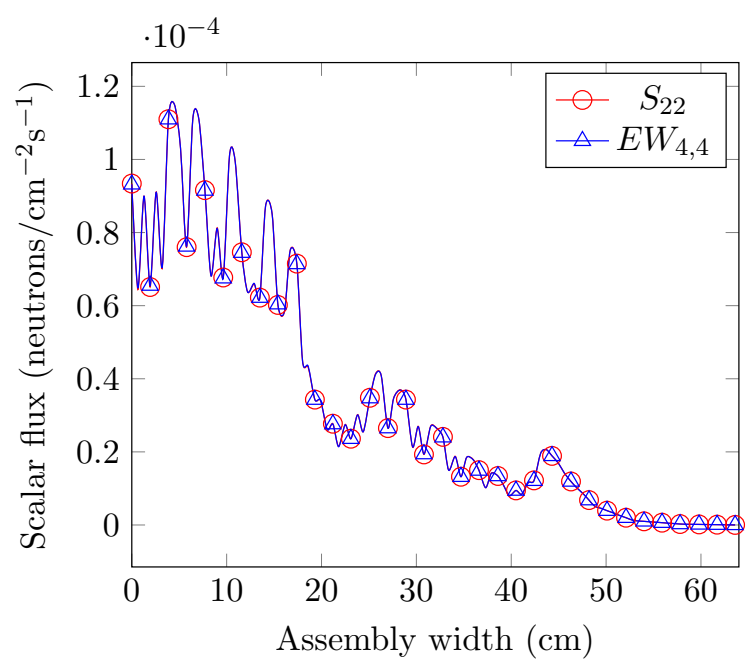

(b) Neutron group 7

Figure 14: Scalar flux values for the C5 MOX assembly problem, computed along the line $(0,0)-(64.26,64.26)$, using the $E W_{4,4}$ and $S_{22}$ discretisations.

Finally, the angular flux plots for the $E W_{4,4}$ and $S_{22}$ discretisations are presented in figures 15 and 16, for various spatial nodes and neutron groups. To investigate the potential benefits from angular adaptivity, three different filtered $E W_{4,4}$ angular flux plots are included, in which the angular flux coefficients below a threshold level have been removed. Filter 1 represents the zeroing out of angular flux coefficients that are smaller than four orders of magnitude of the maximum coefficient, whereas filter 2 and filter 3 represent the zeroing out of angular flux coefficients that are smaller than three and two orders of magnitude respectively.

In figure 15 the angular flux plots for $S_{22}$ and $E W_{4,4}$ are very similar, with small differences attributed to the different quadrature of the angular domain for the two discretisations. Comparing the $E W_{4,4}$ results with the filtered $E W_{4,4}$ results, there is strong evidence in favour of angular adaptivity. At this spatial node, the angular flux plot for $E W_{4,4}$ filter 2 is nearly identical to the $E W_{4,4}$ result, but only uses $36.7 \%$ of the coefficients. Similarly the $E W_{4,4}$ filter 3 result is also very close to the $E W_{4,4}$ result, but only needs $10.9 \%$ of the coefficients. Thus, depending on the desired accuracy, a significant reduction in the angular degrees of freedom can be achieved for this spatial node by using angular adaptivity.

Figure 16 presents a very similar situation. Filter 2 and filter 3 result in $30.1 \%$ and $13.3 \%$ of the angular degrees of freedom, while retaining the quality of the solution. In fact both 
figures 15 and 16 present the ideal scenarios for angular adaptivity. Directional problems where peaks in the angular flux are restricted to small regions of the angular domain can benefit significantly from angular adaptivity, as a large number of the angular degrees of freedom can be removed without degrading the quality of the solution. This means that a reduction of angular degrees of freedom can be achieved for directional areas, while smooth areas with wide angular distributions can retain the full angular expansion. This is a a major advantage compared to most $S_{n}$ schemes, where the same angular resolution is applied at every spatial node.

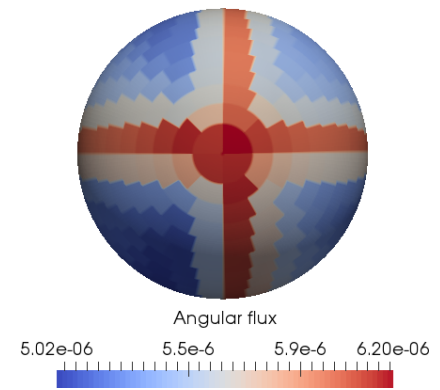

(a) $S_{22}$

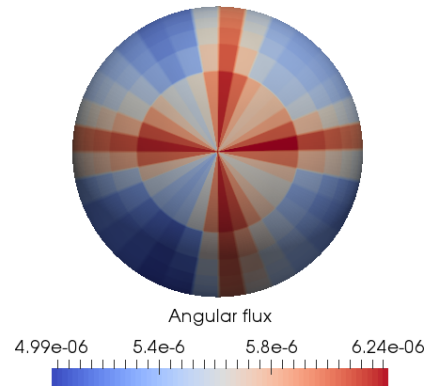

(b) $E W_{4,4}$

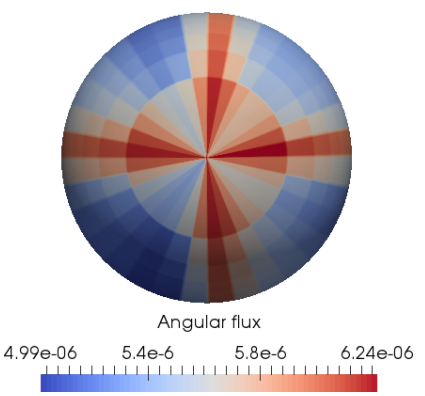

(c) $E W_{4,4}$ filter $1(84.8 \%)$

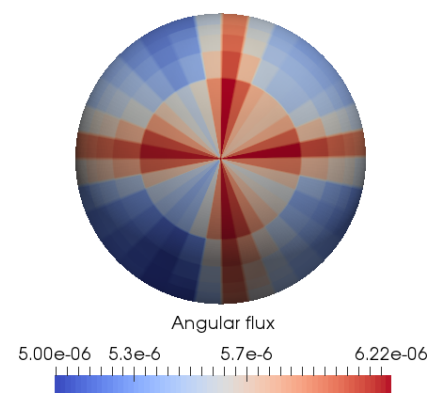

(d) $E W_{4,4}$ filter $2(36.7 \%)$

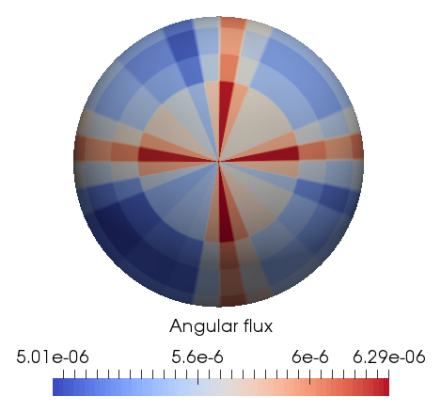

(e) $E W_{4,4}$ filter $3(10.9 \%)$

Figure 15: Neutron group 6 angular flux plots at node $1(10,10)$. For the filter cases, the number in parenthesis denotes the percentage of wavelet coefficients retained. For an $E W_{4,4}$ approximation, there are 256 of such wavelet angular coefficients. The $S_{22}$ approximation uses 264 angular expansion coefficients respectively. For each angular flux plot shown, the $x$ axis is in the horizontal direction relative to the page orientation, the $y$ axis is in the vertical direction relative to the page orientation, and the $z$ axis is in the direction inside the page. The angular flux plot for a fuel pin in the $\mathrm{UO}_{2}$ or MOX region is expected to be isotropic. Note that node $1(10,10)$ however does not directly center on a fuel pin, but rather a location that is in-between two adjacent fuel pins, hence why the plotted angular flux is not isotropic. 


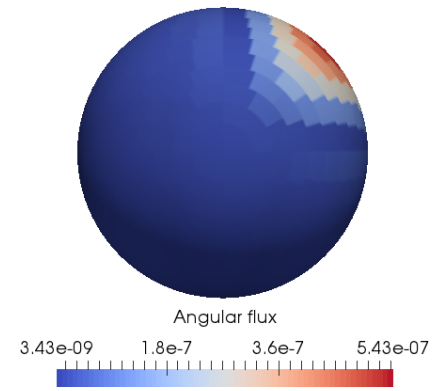

(a) $S_{22}$

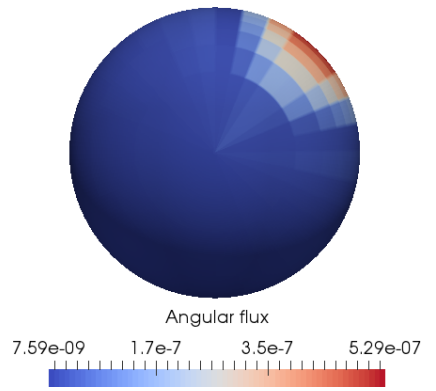

(b) $E W_{4,4}$

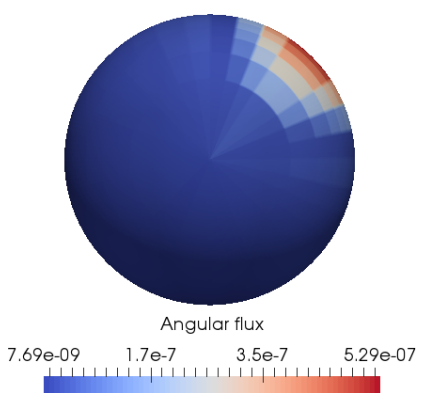

(c) $E W_{4,4}$ filter $1(53.1 \%)$

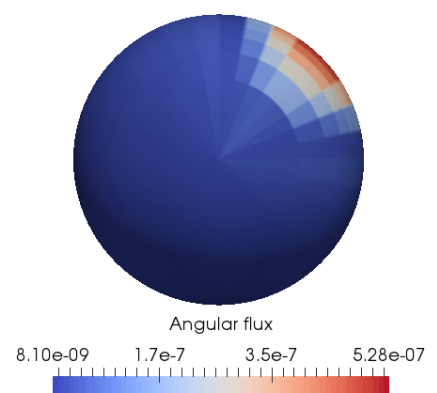

(d) $E W_{4,4}$ filter $2(30.1 \%)$

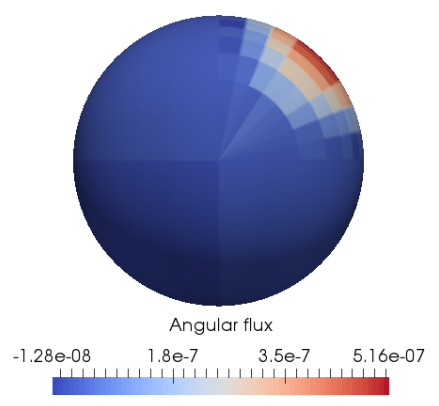

(e) $E W_{4,4}$ filter $3(13.3 \%)$

Figure 16: Neutron group 1 angular flux plots at node $6(50,50)$. For the filter cases, the number in parenthesis denotes the percentage of wavelet coefficients retained. For an $E W_{4,4}$ approximation, there are 256 of such wavelet angular coefficients. The $S_{22}$ approximation uses 264 angular expansion coefficients respectively. For each angular flux plot shown, the $x$ axis is in the horizontal direction relative to the page orientation, the $y$ axis is in the vertical direction relative to the page orientation, and the $z$ axis is in the direction inside the page. As expected, the fast neutrons from this angular flux plot are streaming out away from the reactor. 


\section{Conclusion}

This article has presented a Haar wavelet expansion method for discretising the angular dimension of the Boltzmann transport equation. It was formed from a tensor product of two 1 dimensional Haar wavelet expansions that respectively resolved the azimuthal and polar directions of particle travel. The wavelet method was combined with a subgrid scale finite element formulation for resolving the spatial dependence of the transport equation. This article included a modification of the SGS method that enabled the efficient solving of the Haar wavelet approximations. The stabilization term could be computed in order $\mathcal{M}$ operations, rather than $\mathcal{M}^{3}$, which was essential when large angular expansions were used. This linear growth with angle size makes the Haar wavelet method detailed in this work practical as a discretisation technique for the Boltzmann transport equation.

The numerical examples presented here demonstrated the ability of the wavelets to resolve both shielding and reactor physics problems. The analysis centered on the performance of the wavelets with and without the modification to the SGS formulation, and also on the performance of the wavelets in comparison to the traditional $S_{n}$ scheme. Importantly it was demonstrated that the SGS modification had no detrimental effect in comparison to the original SGS formulation. Therefore large computational efficiency gains were made whilst maintaining stability and accuracy of the solutions.

The wavelet scheme did show slower solution convergence when compared to the $S_{n}$ method using a different quadrature scheme. However this does not take into account the fact that it was not necessary to include the full set of wavelet functions within the approximation. Here it was shown that wavelets could be eliminated from the calculation, if their associated coefficient did not exceed a threshold, with minimal effect on the solution. In many cases over $60 \%$ of wavelets could be removed, and for shielding applications with high streaming this could potentially increase further still. This shows that substantial reduction in problem size can be achieved through an adaptive wavelets approach, and this could easily compensate the slower

convergence and exceed the performance of the traditional $S_{n}$ method. The self-selection of the wavelets by the model itself, and its capabilities in comparison to traditional techniques, will be the topic of a future article. 


\section{Acknowledgments}

We acknowledge the funding of this work through the EPSRC grant refs.: EP/M022684/1 and EP/J002011/1. This work would not have been possible without the help of Atyab Calloo (PhD candidate at Polytechnique Montréal) whose technical suggestions greatly improved the quality of the manuscript.

\section{References}

[1] A. Grossmann and J. Morlet. Decomposition of hardy functions into square integrable wavelets of constant shape. SIAM Journal on Mathematical Analysis, 15(4):723-736, 1984.

[2] I. Daubechies. Orthonormal bases of compactly supported wavelets. Communications on Pure and Applied Mathematics, 41(7):909-996, 1988.

[3] R. Kronland-Martinet, J. Morlet, and A. Grossmann. Analysis of sound patterns through wavelet transforms. International Journal of Pattern Recognition and Artificial Intelligence, 1(2):273-302, 1987.

[4] P. Schroder and W. Sweldens. Spherical wavelets: Efficiently representing functions on the sphere. Proceedings of the 22nd Annual Conference on Computer Graphics and Interactive Techniques, pages 161-172, 1995.

[5] E. Aboufadel and S. Schlicker. Discovering Wavelets. New York: John Wiley \& Sons Inc., 1999.

[6] A.G. Buchan, C.C. Pain, M.D. Eaton, R.P. Smedley-Stevenson, and A.J.H. Goddard. Linear and quadratic octahedral wavelets on the sphere for angular discretisations of the boltzmann transport equation. Annals of Nuclear Energy, 32(11):1224 - 1273, 2005.

[7] B. Li and X. Chen. Wavelet-based numerical analysis: A review and classification. Finite Elements in Analysis and Design, 81:14 - 31, 2014.

[8] K. Schneider and M. Farge. Numerical simulation of a mixing layer in an adaptive wavelet basis. Comptes Rendus de l'Académie des Sciences - Series IIB Mechanics-Physics-Astronomy, 328(3):263 - 269, 2000. 
[9] G.W. Wei, D.S. Zhang, S.C. Althorpe, D.J. Kouri, and D.K. Hoffman. Wavelet-distributed approximating functional method for solving the navier-stokes equation. Computer Physics Communications, 115(1):18 - 24, 1998.

[10] X. Zhou and Y. He. Using divergence free wavelets for the numerical solution of the 2-d stationary navierstokes equations. Applied Mathematics and Computation, 163(2):593 $607,2005$.

[11] M.H. Heydari, M.R. Hooshmandasl, and F. Mohammadi. Legendre wavelets method for solving fractional partial differential equations with dirichlet boundary conditions. Applied Mathematics and Computation, 234:267 - 276, 2014.

[12] S.L. Ho and S.Y. Yang. Wavelet-galerkin method for solving parabolic equations in finite domains. Finite Elements in Analysis and Design, 37(12):1023 - 1037, 2001.

[13] G. Chiavassa, M. Guichaoua, and J. Liandrat. Two adaptive wavelet algorithms for non-linear parabolic partial differential equations. Computers \& Fluids, 31(4-7):467-480, 2002.

[14] M.A. Alves, P. Cruz, A. Mendes, F.D. Magalhaes, F.T. Pinho, and P.J. Oliveira. Adaptive multiresolution approach for solution of hyperbolic pdes. Computer Methods in Applied Mechanics and Engineering, 191(36):3909 - 3928, 2002.

[15] T. Hong and B.L.N. Kennett. On a wavelet-based method for the numerical simulation of wave propagation. Journal of Computational Physics, 183(2):577 - 622, 2002.

[16] S.R. Massel. Wavelet analysis for processing of ocean surface wave records. Ocean Engineering, 28(8):957 - 987, 2001.

[17] S.M. Reckinger, O.V. Vasilyev, and B. Fox-Kemper. Adaptive wavelet collocation method on the shallow water model. Journal of Computational Physics, 271:342 - 359, 2014.

[18] A.G. Buchan, S.R. Merton, C.C. Pain, and R.P. Smedley-Stevenson. Riemann boundary conditions for the boltzmann transport equation using arbitrary angular approximations. Annals of Nuclear Energy, 38(5):1186 - 1195, 2011.

[19] L. Cao, H. Wu, and Y. Zheng. Solution of neutron transport equation using daubechies' wavelet expansion in the angular discretization. Nuclear Engineering and Design, 238 (9):2292 - 2301, 2008.

[20] Y. Zheng, H. Wu, and L. Cao. Wavelet-based angular dependence analysis of the heterogeneous calculation on mox fuel lattice. Nuclear Engineering and Design, 239 (10):1804 - 1810, 2009. 
[21] Y. Zheng, H. Wu, and L. Cao. An improved three-dimensional wavelet-based method for solving the first-order boltzmann transport equation. Annals of Nuclear Energy, 36 (9):1440 - 1449, 2009.

[22] A. Adam, A.G. Buchan, M.D. Piggott, C.C. Pain, J. Hill, and M.A. Goffin. Adaptive haar wavelets for the angular discretisation of spectral wave models. Journal of Computational Physics, 305:521 - 538, 2016.

[23] W. Freeden and U. Windheuser. Combined spherical harmonic and wavelet expansiona future concept in earth's gravitational determination. Applied and Computational Harmonic Analysis, 4(1):1 - 37, 1997.

[24] L. Demanet, L. Jacques, P. Vandergheynst, and J.P. Antoine. Wavelets on the sphere: implementation and approximations. Applied and Computational Harmonic Analysis, 13 (3):177 - 200, 2002.

[25] J.P. Antoine and P Vandergheynst. Wavelets on the 2-sphere: A group-theoretical approach. Applied and Computational Harmonic Analysis, 7(3):262 - 291, 1999.

[26] A.G. Buchan, C.C. Pain, M.D. Eaton, R.P. Smedley-Stevenson, and A.J.H. Goddard. Self-adaptive spherical wavelets for angular discretizations of the boltzmann transport equation. Nuclear Science and Engineering, 158(3):244-263, 2008.

[27] A. Patra and S.S. Ray. Two-dimensional haar wavelet collocation method for the solution of stationary neutron transport equation in a homogeneous isotropic medium. Annals of Nuclear Energy, 70:30 - 35, 2014.

[28] A. Patra and S.S. Ray. A numerical approach based on haar wavelet operational method to solve neutron point kinetics equation involving imposed reactivity insertions. Annals of Nuclear Energy, 68:112 - 117, 2014.

[29] M. L. Adams and E. W. Larsen. Fast iterative methods for discrete-ordinates particle transport calculations. Progress in Nuclear Energy, 40(1):3 - 159, 2002.

[30] J. P. Jessee, W. A. Fiveland, L. H. Howell, P. C. Colella, and R. B. Pember. An adaptive mesh refinement algorithm for the radiative transport equation. Journal of Computational Physics, 139(2):380 - 398, 1998.

[31] J. C. Ragusa and Y. Wang. A two-mesh adaptive mesh refinement technique for sn neutral-particle transport using a higher-order dgfem. Journal of Computational and Applied Mathematics, 233(12):3178 - 3188, 2010.

[32] A.G. Buchan, A.A. Calloo, M.G. Goffin, S. Dargaville, F. Fang, C.C. Pain, and I.M. Navon. A pod reduced order model for resolving angular direction in neutron/photon transport problems. Journal of Computational Physics, 296:138-157, 2015. 
[33] M.A. Goffin, C.M.J. Baker, A.G. Buchan, C.C. Pain, M.D. Eaton, and P.N. Smith. Minimising the error in eigenvalue calculations involving the boltzmann transport equation using goal-based adaptivity on unstructured meshes. Journal of Computational Physics, 242:726 - 752, 2013.

[34] A.G. Buchan, A.S. Candy, S.R. Merton, C.C. Pain, J.I. Hadi, M.D. Eaton, A.J.H. Goddard, R.P. Smedley-Stevenson, and G.J. Pearce. The inner-element subgrid scale finite element method for the boltzmann transport equation. Nuclear Science and Engineering, 164(2):105 - 121, 2010.

[35] A.G. Buchan and C.C. Pain. An efficient space-angle subgrid scale discretisation of the neutron transport equation. Submitted to Annals of Nuclear Energy, 2014.

[36] D. Kershaw and J. Harte. 2d deterministic radiation transport with the discontinuous finite element method. Technical report, Lawrence Livermore National Lab., 1993.

[37] A.G. Buchan, C.C. Pain, A.P. Umpleby, and R.P. Smedley-Stevenson. A sub-grid scale finite element agglomeration multigrid method with application to the boltzmann transport equation. International Journal for Numerical Methods in Engineering, 92(3): 318-342, October 2012. ISSN 1097-0207.

[38] A.M. Watson. The WN adaptive method for numerical solution of particle transport problems. Texas A\&M University, April 2006.

[39] C. Cavarec, J. F. Perronand, D. Verwaerde, and J. P. West. The oecd/nea benchmark calculations of power distributions within assemblies. Technical report, Electricité de France, September 1994.

[40] S. Cathalau, J.C. Lefebvre, and J.P. West. Proposal for a second stage of the benchmark on power distributions within assemblies. Technical report, OECD/NEA, April 1996. An earlier version of the published OECD/NEA benchmark. 